Pacific

Journal of

Mathematics

THE MODULI OF FLAT PU(2,1) STRUCTURES ON RIEMANN SURFACES

Eugene Z. XiA 


\title{
THE MODULI OF FLAT PU(2,1) STRUCTURES ON RIEMANN SURFACES
}

\author{
Eugene Z. XiA
}

For a compact Riemann surface $X$ of genus $g>1$, $\operatorname{Hom}\left(\pi_{1}(X), \mathrm{PU}(p, q)\right) / \mathrm{PU}(p, q)$ is the moduli space of flat $\mathrm{PU}(p, q)$-connections on $X$. There are two integer invariants, $d_{P}, d_{Q}$, associated with each $\sigma \in \operatorname{Hom}\left(\pi_{1}(X), \mathrm{PU}(p, q)\right) /$ $\mathrm{PU}(p, q)$. These invariants are related to the Toledo invariant $\tau$ by $\tau=2 \frac{q d_{P}-p d_{Q}}{p+q}$. This paper shows, via the theory of Higgs bundles, that if $q=1$, then $-2(g-1) \leq \tau \leq 2(g-1)$. Moreover, $\operatorname{Hom}\left(\pi_{1}(X), \mathrm{PU}(2,1)\right) / \mathrm{PU}(2,1)$ has one connected component corresponding to each $\tau \in \frac{2}{3} \mathbb{Z}$ with $-2(g-1) \leq$ $\tau \leq 2(g-1)$. Therefore the total number of connected components is $6(g-1)+1$.

\section{Introduction.}

Let $X$ be a smooth projective curve over $\mathbb{C}$ with genus $g>1$. The deformation space

$$
\mathbb{C N}_{B}=\operatorname{Hom}^{+}\left(\pi_{1}(X), \operatorname{PGL}(n, \mathbb{C})\right) / \operatorname{PGL}(n, \mathbb{C})
$$

is the space of equivalence classes of semi-simple $\operatorname{PGL}(n, \mathbb{C})$-representations of the fundamental group $\pi_{1}(X)$. This is the $\operatorname{PGL}(n, \mathbb{C})$-Betti moduli space on $X[\mathbf{2 2}, \mathbf{2 3}, \mathbf{2 4}]$. A theorem of Corlette, Donaldson, Hitchin and Simpson relates $\mathbb{C} \mathcal{N}_{B}$ to two other moduli spaces, $\mathbb{C} \mathcal{N}_{D R}$ and $\mathbb{C} \mathcal{N}_{\text {Dol }}$ - the $\operatorname{PGL}(n, \mathbb{C})$ de Rham and the PGL $(n, \mathbb{C})$-Dolbeault moduli spaces, respectively $[\mathbf{3}, \mathbf{5}, \mathbf{1 1}$, 21]. The Dolbeault moduli space consists of holomorphic objects (Higgs bundles) over $X$; therefore, the classical results of analytic and algebraic geometry can be applied to the study of the Dolbeault moduli space.

Since $\operatorname{PU}(p, q) \subset \operatorname{PGL}(n, \mathbb{C}), \mathbb{C N}_{B}$ contains the space

$$
\mathcal{N}_{B}=\operatorname{Hom}^{+}\left(\pi_{1}(X), \mathrm{PU}(p, q)\right) / \mathrm{PU}(p, q) .
$$

The space $\mathcal{N}_{B}$ will be referred to as the $\mathrm{PU}(p, q)$-Betti moduli space which similarly corresponds to some subspaces $\mathcal{N}_{D R}$ and $\mathcal{N}_{\text {Dol }}$ of $\mathbb{C} \mathcal{N}_{D R}$ and $\mathbb{C} \mathcal{N}_{\text {Dol }}$, respectively. We shall refer to $\mathcal{N}_{D R}$ and $\mathcal{N}_{\text {Dol }}$ as the $\mathrm{PU}(p, q)$-de Rham and the $\mathrm{PU}(p, q)$-Dolbeault moduli spaces.

The Betti moduli spaces are of great interest in the field of geometric topology and uniformization. In the case of $p=q=1$, Goldman analyzed 
$\mathcal{N}_{B}$ and determined the number of its connected components to be $4 g-3$ [6]. Hitchin subsequently considered $\mathcal{N}_{\text {Dol }}$ in the case of $p=q=1$ and determined its topology [11].

In this paper, we analyze $\mathcal{N}_{\text {Dol }}$ for the case of $p=2, q=1$ and determine its number of connected components. In addition, we produce a new algebraic proof, via the Higgs-bundle theory, of a theorem by Toledo on the bounds of the Toledo invariant $[\mathbf{2 6}, \mathbf{2 7}]$.

An element $\sigma \in \operatorname{Hom}^{+}\left(\pi_{1}(X), \mathrm{PU}(p, q)\right)$ defines a flat principal $\mathrm{PU}(p, q)$ bundle $P$ over $X$. Such a flat bundle may be lifted to a principal $\mathrm{U}(p, q)$ bundle $\hat{P}$ with a Yang-Mills connection $D[\mathbf{2}, \mathbf{3}, \mathbf{5}, \mathbf{1 1}, \mathbf{2 1}]$. Let $E$ be the rank- $(p+q)$ vector bundle associated with $(\hat{P}, D)$. The second cohomology $\mathrm{H}^{2}(X, \mathbb{Z})$ is isomorphic to $\mathbb{Z}$, so one may identify the Chern class $c_{1}(E) \in$ $\mathrm{H}^{2}(X, \mathbb{Z})$ with an integer, the degree of $E$. Suppose we impose the additional condition

$$
0 \leq \operatorname{deg}(E)<n
$$

Then the above construction gives rise to a unique obstruction class $o_{2}(E) \in$ $\mathrm{H}^{2}\left(X, \pi_{1}(\mathrm{U}(p, q))\right)[25]$. The obstruction class is invariant under the conjugation action of $\mathrm{PU}(p, q)$; therefore, one obtains the obstruction map:

$$
o_{2}: \operatorname{Hom}^{+}\left(\pi_{1}(X), \mathrm{PU}(p, q)\right) / \mathrm{PU}(p, q) \longrightarrow \mathrm{H}^{2}\left(X, \pi_{1}(\mathrm{U}(p, q))\right) \cong \mathbb{Z} \times \mathbb{Z} .
$$

The maximum compact subgroup of $\mathrm{U}(p, q)$ is $\mathrm{U}(p) \times \mathrm{U}(q)$. Hence topologically $E$ is a direct sum $E_{P} \oplus E_{Q}$ with

$$
\operatorname{deg}(E)=\operatorname{deg}\left(E_{P}\right)+\operatorname{deg}\left(E_{Q}\right) .
$$

The obstruction class $o_{2}(E)$ is then $\left(\operatorname{deg}\left(E_{P}\right), \operatorname{deg}\left(E_{Q}\right)\right) \in \mathbb{Z} \times \mathbb{Z}$. Associated with $\sigma$ is the Toledo invariant $\tau$ which relates to $d_{P}=\operatorname{deg}\left(E_{P}\right)$ and $d_{Q}=$ $\operatorname{deg}\left(E_{Q}\right)$ by the formula $[\mathbf{7}, \mathbf{2 6}, \mathbf{2 7}]$

$$
\tau=2 \frac{\operatorname{deg}\left(E_{P} \otimes E_{Q}^{*}\right)}{p+q}=2 \frac{q d_{P}-p d_{Q}}{p+q} .
$$

This explains why the Toledo invariant of a $\mathrm{PU}(2,1)$ representation cannot be an odd integer [7]. The main result presented here is the following:

Theorem 1.1. $\operatorname{Hom}^{+}\left(\pi_{1}(X), \mathrm{PU}(2,1)\right) / \mathrm{PU}(2,1)$ has one connected component for each $\tau \in \frac{2}{3} \mathbb{Z}$ with $-2(g-1) \leq \tau \leq 2(g-1)$. Therefore the total number of connected components is $6(g-1)+1$.

We shall also provide a new proof en route to the following theorem:

Theorem 1.2 (Toledo). Suppose $\sigma \in \operatorname{Hom}^{+}\left(\pi_{1}(X), \mathrm{PU}(p, 1)\right)$ and $\tau$ is the Toledo class of $\sigma$. Then

$$
-2(g-1) \leq \tau \leq 2(g-1) .
$$

Moreover $\tau= \pm 2(g-1)$ implies $\sigma$ is reducible. 
These results are related to the results of Domic and Toledo $[\mathbf{4}, \mathbf{2 6}, \mathbf{2 7}]$ and, as being pointed out to the author recently, are also related to the work of Gothen [8] which computed the Poincaré polynomials for the components of $\operatorname{Hom}\left(\pi_{1}(X), \operatorname{PSL}(3, \mathbb{C})\right) / \operatorname{PSL}(3, \mathbb{C})$, where $\operatorname{deg}(E)$ is coprime to 3 .

\section{Acknowledgments.}

Most of this research was carried out while the author was at the University of Maryland at College Park. I thank J. Adams, K. Coombes, P. Green, K. Joshi, S. Kudla, P. Newstead, J. Poritz and especially W. Goldman and C. Simpson for insightful discussions over the course of the research. I thank the referee for helpful suggestions.

\section{Backgrounds and Preliminaries.}

In this section, we briefly outline the constructions of the Betti, de Rham and Dolbeault moduli spaces. For details, see $[\mathbf{2}, \mathbf{3}, \mathbf{5}, \mathbf{1 1}, \mathbf{1 2}, \mathbf{1 8}, \mathbf{2 1}, \mathbf{2 2}$, 23, 24].

2.1. The Betti Moduli Space. The fundamental group $\pi_{1}(X)$ is generated by $S=\left\{A_{i}, B_{i}\right\}_{i=1}^{g}$, subject to the relation

$$
\prod_{i=1}^{g} A_{i} B_{i} A_{i}^{-1} B_{i}^{-1}=e .
$$

Denote by I and $[\mathrm{I}]$ the identities of $\operatorname{GL}(n, \mathbb{C})$ and $\operatorname{PGL}(n, \mathbb{C})$, respectively. Define

$$
\begin{gathered}
R: \operatorname{PGL}(n, \mathbb{C})^{2 g} \longrightarrow \operatorname{PGL}(n, \mathbb{C}) \\
\mathcal{R}: \operatorname{GL}(n, \mathbb{C})^{2 g} \longrightarrow \operatorname{GL}(n, \mathbb{C})
\end{gathered}
$$

to be the commutator maps:

$$
\left(X_{1}, Y_{1}, \ldots, X_{g}, Y_{g}\right) \stackrel{R, \mathcal{R}}{\longrightarrow} \prod_{i=1}^{g} X_{i} Y_{i} X_{i}^{-1} Y_{i}^{-1} .
$$

The group

$$
\left\{\zeta \mathrm{I}: \zeta \in \mathbb{C}, \zeta^{n}=1\right\}
$$

is isomorphic to $\mathbb{Z}_{n}$. The space $\mathcal{R}^{-1}\left(\mathbb{Z}_{n}\right)$ is identified with the representation space $\operatorname{Hom}(\Gamma, \operatorname{GL}(n, \mathbb{C}))$, where $\Gamma$ is the central extension $[\mathbf{2}, \mathbf{1 1}]$ :

$$
0 \longrightarrow \mathbb{Z}_{n} \longrightarrow \Gamma \longrightarrow \pi_{1}(X) \longrightarrow 0 \text {. }
$$

Each element $\rho \in \mathcal{R}^{-1}\left(\mathbb{Z}_{n}\right)$ acts on $\mathbb{C}^{n}$ via the standard representation of $\mathrm{GL}(n, \mathbb{C})$. The representation $\rho$ is called reducible (irreducible) if its action on $\mathbb{C}^{n}$ is reducible (irreducible). A representation $\rho$ is called semi-simple if it is a direct sum of irreducible representations. Let $\zeta_{1}=e^{2 \pi \mathrm{i} / n}$ and define

$$
\mathbb{C} \mathcal{M}_{B}(c)=\left\{\sigma \in \mathcal{R}^{-1}\left(\zeta_{1}^{c} \mathrm{I}\right): \sigma \text { is semi-simple }\right\} / \operatorname{GL}(n, \mathbb{C}),
$$




$$
\begin{gathered}
\mathbb{C M}_{B}=\bigcup_{c=0}^{n-1} \mathbb{C} \mathcal{M}_{B}(c), \\
\mathbb{C N}_{B}(c)=\mathbb{C M}_{B}(c) / \operatorname{Hom}\left(\pi_{1}(X), \mathbb{C}^{*}\right) \\
=\operatorname{Hom}^{+}\left(\pi_{1}(X), \operatorname{PGL}(n, \mathbb{C})\right) / \operatorname{PGL}(n, \mathbb{C}) .
\end{gathered}
$$

Fix $p, q$ such that $p+q=n$. Denote by $\mathcal{R}_{U}$ the restriction of $\mathcal{R}$ to the subgroup $\mathrm{U}(p, q)^{2 g}$. Define

$$
\begin{gathered}
\mathcal{M}_{B}(c)=\left\{\sigma \in \mathcal{R}_{U}^{-1}\left(\zeta_{1}^{c} \mathrm{I}\right): \sigma \text { is semi-simple }\right\} / \mathrm{U}(p, q), \\
\mathcal{M}_{B}=\bigcup_{c=0}^{n-1} \mathcal{M}_{B}(c) .
\end{gathered}
$$

Note the center of $\mathrm{U}(p, q)$ is $\mathrm{U}(1)$ and is contained in the center of $\operatorname{GL}(n, \mathbb{C})$. It follows that $\mathcal{M}_{B}(c) \subset \mathbb{C} \mathcal{M}_{B}(c)$. Define

$$
\begin{gathered}
\mathcal{N}_{B}(c)=\mathcal{M}_{B}(c) / \operatorname{Hom}\left(\pi_{1}(X), \mathrm{U}(1)\right) \\
\mathcal{N}_{B}=\mathcal{M}_{B} / \operatorname{Hom}\left(\pi_{1}(X), \mathrm{U}(1)\right)=\operatorname{Hom}^{+}\left(\pi_{1}(X), \mathrm{U}(p, q)\right) / \mathrm{U}(p, q) .
\end{gathered}
$$

All the spaces constructed here that contain the symbols $\mathcal{M}_{B}$ or $\mathcal{N}_{B}$ will be loosely referred to as Betti moduli spaces. The subspace of irreducible elements of a Betti moduli space will be denoted by an $s$ superscript. For example, $\mathbb{C} \mathcal{M}_{B}^{s}$ denotes the subspace of irreducible elements of $\mathbb{C} \mathcal{M}_{B}$.

2.2. The de Rham Moduli Space. Suppose $P$ is a principal $\operatorname{GL}(n, \mathbb{C})$ bundle on $X, E$ its associated vector bundle of rank $n$ and $\mathcal{G}_{\mathbb{C}}(E)$ the group of $\operatorname{GL}(n, \mathbb{C})$-gauge transformations on $E$. A connection is called Yang-Mills (or central) if its curvature is central [2]. The gauge group $\mathcal{G}_{\mathbb{C}}(E)$ acts on the space of $\operatorname{GL}(n, \mathbb{C})$-connections on $E$ and preserves the subspace of Yang-Mills connections. Fix $E$ with $\operatorname{deg}(E)=c$. The de Rham moduli space $\mathbb{C M}_{D R}(c)$ on $E$ is defined to be the $\mathcal{G}_{\mathbb{C}}(E)$-equivalence classes of Yang-Mills connections.

Let $\mathcal{M}_{D R}(c)$ denote the space of $\mathrm{U}(p, q)$-gauge equivalence classes of $\mathrm{U}(p, q)$-central connections on $E$. In other words, $\mathcal{M}_{D R}(c)$ is constructed as $\mathbb{C} \mathcal{M}_{D R}(c)$, but with $\mathrm{U}(p, q)$ replacing $\mathrm{GL}(n, \mathbb{C})$. Since the center of $\mathrm{U}(p, q)$ is contained in the center of $\mathrm{GL}(n, \mathbb{C}), \mathcal{M}_{D R}(c) \subset \mathbb{C} \mathcal{M}_{D R}(c)$.

The space of $\mathbb{C}^{*}$-gauge equivalence classes of $\mathbb{C}^{*}$-connections on $X$ is $\mathrm{H}^{1}\left(X, \mathbb{C}^{*}\right)$ which acts on $\mathbb{C} \mathcal{M}_{D R}(c)[2]$. Denote the quotient $\mathbb{C N}_{D R}(c)$. This action corresponds to the action of $\operatorname{Hom}\left(\pi_{1}(X), \mathbb{C}^{*}\right)$ on $\mathbb{C} \mathcal{M}_{B}(c)$ and the quotient $\mathbb{C N}_{D R}(c)$ corresponds to $\mathbb{C N}_{B}(c)$. Similarly, the space of U(1)gauge equivalence classes of $\mathrm{U}(1)$-connections on $X$ is $\mathrm{H}^{1}(X, \mathrm{U}(1))$ which acts on $\mathcal{M}_{D R}(c)$ and the quotient is denoted by $\mathcal{N}_{D R}(c)$. Define

$$
\mathbb{C} \mathcal{M}_{D R}=\bigcup_{c=-\infty}^{\infty} \mathbb{C} \mathcal{M}_{D R}(c), \quad \mathbb{C N}_{D R}=\bigcup_{c=-\infty}^{\infty} \mathbb{C} \mathcal{N}_{D R}(c)
$$




$$
\mathcal{M}_{D R}=\bigcup_{c=-\infty}^{\infty} \mathcal{M}_{D R}(c), \quad \mathcal{N}_{D R}=\bigcup_{c=-\infty}^{\infty} \mathcal{N}_{D R}(c) .
$$

All the spaces constructed here that contain the symbols $\mathcal{M}_{D R}$ or $\mathcal{N}_{D R}$ will be loosely referred to as de Rham moduli spaces. A central connection is irreducible if $(E, D)=\left(E_{1} \oplus E_{2}, D_{1} \oplus D_{2}\right)$ implies $\operatorname{rank}\left(E_{1}\right)=0$ or $\operatorname{rank}\left(E_{2}\right)=0$. The subspace of irreducible elements of a de Rham moduli space will be denoted by an $s$ superscript.

Theorem 2.1. The moduli space $\mathbb{C M}_{B}(c)$ is homeomorphic to $\mathbb{C} \mathcal{M}_{D R}(c)$.

Proof. See $[\mathbf{3}, \mathbf{5}, \mathbf{1 1}]$.

Consider all the objects we have defined so far with subscripts $B$ or $D R$. With Theorem 2.1, one can verify the following: Suppose two objects have subscripts $B$ or $D R$. Then the two objects are homeomorphic if they only differ in subscripts. For example, $\mathcal{N}_{B}(c)$ is homeomorphic to $\mathcal{N}_{D R}(c)$.

Since the maximum compact subgroup of $\mathrm{U}(p, q)$ is $\mathrm{U}(p) \times \mathrm{U}(q),(E, D) \in$ $\mathcal{M}_{D R}$ implies $E$ is a direct sum of a $\mathrm{U}(p)$ and a $\mathrm{U}(q)$-bundle:

$$
E=E_{p} \oplus E_{q},
$$

where the ranks of $E_{p}$ and $E_{q}$ are $p$ and $q$, respectively. Therefore, associated to each $(E, D)$ are the invariants

$$
d_{P}=\operatorname{deg}\left(E_{P}\right) \text { and } d_{Q}=\operatorname{deg}\left(E_{Q}\right),
$$

with

$$
d_{P}+d_{Q}=\operatorname{deg}(E)=c .
$$

The Toledo invariant $\tau$ is $[\mathbf{7}, \mathbf{2 6}, \mathbf{2 7}]$

$$
\tau=2 \frac{\operatorname{deg}\left(E_{P} \otimes E_{Q}^{*}\right)}{n}=2 \frac{q d_{P}-p d_{Q}}{n} .
$$

The subspace of $\mathcal{M}_{D R}(c)$ with a fixed Toledo invariant $\tau$ is denoted by $\mathcal{M}_{D R}^{\tau}$. By the equivalence of Betti and de Rham moduli spaces, one may define the Toledo invariant on $\mathcal{M}_{B}(c)$. Denote by $\mathcal{M}_{B}^{\tau}$ the subspace of $\mathcal{M}_{B}(c)$ with a fixed Toledo invariant $\tau$. The $\mathrm{H}^{1}(X, \mathrm{U}(1))$ action on $\mathcal{M}_{D R}(c)$ preserves $\mathcal{M}_{D R}^{\tau}$ and the quotient is denoted by $\mathcal{N}_{D R}^{\tau}$. In the Betti moduli space, the $\operatorname{Hom}\left(\pi_{1}(X), \mathrm{U}(1)\right)$ action on $\mathcal{M}_{B}$ preserves $\mathcal{M}_{B}^{\tau}$, and the quotient is denoted by $\mathcal{N}_{B}^{\tau}$.

2.3. The Dolbeault Moduli Space. Let $E$ be a rank $n$ complex vector bundle over $X$ with $\operatorname{deg}(E)=c$. Denote by $\Omega$ the canonical bundle on $X$. A holomorphic structure $\bar{\partial}$ on $E$ induces holomorphic structures on the bundles $\operatorname{End}(E)$ and $\operatorname{End}(E) \otimes \Omega$. A Higgs bundle is a pair $\left(E_{\bar{\partial}}, \Phi\right)$, where $\bar{\partial}$ is a holomorphic structure on $E$ and $\Phi \in \mathrm{H}^{0}\left(X, \operatorname{End}\left(E_{\bar{\partial}}\right) \otimes \Omega\right)$. Such a $\Phi$ is called a Higgs field. We denote the holomorphic bundle $E_{\bar{\partial}}$ by $V$. 
Define the slope of a Higgs bundle $(V, \Phi)$ to be

$$
s(V)=\operatorname{deg}(V) / \operatorname{rank}(V) .
$$

For a fixed $\Phi$, a holomorphic subbundle $W \subset V$ is said to be $\Phi$-invariant if $\Phi(W) \subset W \otimes \Omega$. A pair $(V, \Phi)$ is stable (semi-stable) if $W \subset V$ is $\Phi$-invariant implies

$$
s(W)<(\leq) s(V) .
$$

A Higgs bundle is called poly-stable if it is a direct sum of stable Higgs bundles of the same slope $[\mathbf{1 1}, \mathbf{2 2}]$.

The gauge group $\mathcal{G}_{\mathbb{C}}(E)$ acts on holomorphic structures by pull-back and on Higgs fields by conjugation. Moreover the $\mathcal{G}_{\mathbb{C}}(E)$ action preserves stability, poly-stability and semi-stability. The Dolbeault moduli space $\mathbb{C} \mathcal{M}_{\text {Dol }}(c)$ on $E$ (with $\operatorname{deg}(E)=c$ ), is the $\mathcal{G}_{\mathbb{C}}(E)$-equivalence classes of poly-stable (or $S$-equivalence classes of semi-stable [18]) Higgs bundles $(V, \Phi)$ on $X$ $[11,12,18,22]$. A Higgs bundle is called reducible if it is poly-stable but not stable. Let

$$
\mathbb{C} \mathcal{M}_{\text {Dol }}=\bigcup_{c=-\infty}^{\infty} \mathbb{C} \mathcal{M}_{\text {Dol }}(c) \text {. }
$$

If $D \in \mathbb{C M}_{D R}(c)$, then for any Hermitian metric $h$ on $E$, there is a decomposition,

$$
D=D_{A}+\Psi,
$$

where $D_{A}$ is compatible with $h$ and $\Psi$ is a 1 -form with coefficients in $\mathfrak{p}$. The $(0,1)$ part of $D_{A}$ determines a holomorphic structure $\bar{\partial}_{A}$ on $E$ while the $(1,0)$ part of $\Psi$ is a section of the bundle $\operatorname{End}(E) \otimes \Omega$. There exists a metric $h$ such that the pair

$$
(V, \Phi)=\left(E_{\bar{\partial}_{A}}, \Psi^{1,0}\right)
$$

so constructed is a poly-stable Higgs bundle $[\mathbf{1 1}, \mathbf{2 1}, \mathbf{2 2}]$. Therefore this construction gives a map

$$
f: \mathbb{C} \mathcal{M}_{D R}(c) \longrightarrow \mathbb{C} \mathcal{M}_{\mathrm{Dol}}(c) .
$$

Theorem 2.2 (Corlette, Donaldson, Hitchin, Simpson). The map $f$ is a homeomorphism.

Proof. See $[\mathbf{3}, \mathbf{5}, \mathbf{1 1}, \mathbf{2 1}]$.

\section{The $\mathrm{U}(p, q)$-Yang-Mills Connections.}

Assume $p \geq q$ and $p+q=n$. From the previous section, we know that $\mathcal{M}_{D R} \subset \mathbb{C} \mathcal{M}_{D R}$. Let $D \in \mathbb{C} \mathcal{M}_{D R}(c)$ be a $\operatorname{GL}(n, \mathbb{C})$-Yang-Mills connection on a rank $n$ vector bundle

$$
E \longrightarrow X \text {. }
$$


Proposition 3.1. $D$ is a $\mathrm{U}(p, q)$-Yang-Mills connection if and only if its corresponding Higgs bundle $(V, \Phi) \in \mathbb{C} \mathcal{M}_{\text {Dol }}(c)$ satisfies the following two conditions:

1) $V$ is decomposable into a direct sum:

$$
V=V_{P} \oplus V_{Q}
$$

where $V_{P}, V_{Q}$ are of rank $p, q$, respectively.

2) The Higgs field decomposes into two maps:

$$
\begin{aligned}
& \Phi_{1}: V_{P} \longrightarrow V_{Q} \otimes \Omega, \\
& \Phi_{2}: V_{Q} \longrightarrow V_{P} \otimes \Omega .
\end{aligned}
$$

Proof. Suppose $D$ is a $\mathrm{U}(p, q)$-Yang-Mills connection. Denote by $h$ the Hermitian-Yang-Mills metric on $(E, D)$. Then $D$ decomposes as

$$
D=D_{A}+\Psi,
$$

where $D_{A}$ is the part compatible with $h$. The Cartan decomposition $(\mathfrak{g}=$ $\mathfrak{k} \oplus \mathfrak{p})$ for $\mathfrak{u}(p, q)$ is

$$
\mathfrak{u}(p, q)=(\mathfrak{u}(p) \oplus \mathfrak{u}(q)) \oplus \mathfrak{p} .
$$

If we take the standard representation of $\mathfrak{u}(p, q)$, then elements in $\mathfrak{k}$ are of the form

$$
\left(\begin{array}{ll}
a & 0 \\
0 & d
\end{array}\right)
$$

where $a \in \mathfrak{u}(p), b \in \mathfrak{u}(q)$, respectively. The elements in $\mathfrak{p}$ are then of the form

$$
\left(\begin{array}{ll}
0 & b \\
c & 0
\end{array}\right)
$$

where $b \in \operatorname{Hom}\left(\mathbb{C}^{q}, \mathbb{C}^{p}\right), c \in \operatorname{Hom}\left(\mathbb{C}^{p}, \mathbb{C}^{q}\right)$, respectively. Hence on local charts, $D_{A}$ and $\Psi$ have coefficients in $\mathfrak{k}$ and $\mathfrak{p}$, respectively. In particular, the connection $D_{A}$ is reducible.

The Higgs bundle corresponding to $D$ is $\left(E_{\bar{\partial}_{A}}, \Phi\right)$ where $\bar{\partial}_{A}$ is the $(0,1)$ part of $D_{A}$ and $\Phi$, the $(1,0)$-part of $\Psi$, is considered as a holomorphic bundle map:

$$
\Phi: V \longrightarrow V \otimes \Omega .
$$

Since $D_{A}$ has coefficient in $\mathfrak{k}$, the holomorphic structure on $\mathrm{V}$ defined by $D_{A}^{0,1}$ is a direct sum:

$$
V=V_{P} \oplus V_{Q} .
$$

Since $\Psi$ is block off-diagonal, $\Phi$ is also block off-diagonal implying $\Phi$ can be decomposed into two maps:

$$
\begin{aligned}
& \Phi_{1}: V_{P} \longrightarrow V_{Q} \otimes \Omega, \\
& \Phi_{2}: V_{Q} \longrightarrow V_{P} \otimes \Omega .
\end{aligned}
$$

This proves the only if part of the proposition. 
Suppose $(V, \Phi)$ is a Higgs bundle that satisfies the two conditions of Proposition 3.1. Let $\alpha$ be the constant gauge

$$
\alpha=\left(\begin{array}{cc}
\mathrm{I}_{p} & 0 \\
0 & -\mathrm{I}_{q}
\end{array}\right),
$$

where $\mathrm{I}_{p}, \mathrm{I}_{q}$ are $p \times p, q \times q$ identity matrices, respectively. Then $\alpha$ acts on the space of holomorphic structures on $E$ and fixes $V$. Moreover,

$$
\alpha \Phi \alpha^{-1}=-\Phi
$$

since $\Phi$ is of the form

$$
\Phi=\left(\begin{array}{cc}
0 & \Phi_{1} \\
\Phi_{2} & 0
\end{array}\right) .
$$

Hence by a theorem of Simpson, the corresponding Hermitian-Yang-Mills metric $h$ is invariant under the action of $\alpha$ [21]. In other words, on local charts, $h$ is a Hermitian matrix of the form

$$
h=\left(\begin{array}{ll}
a & 0 \\
0 & d
\end{array}\right),
$$

where $a, d$ are Hermitian matrices of dimension $p \times p, q \times q$, respectively. Hence the corresponding Yang-Mills connection is

$$
D=D_{A}+\Phi+\Phi^{\ddagger},
$$

where $\Phi^{\ddagger}$ is the adjoint of $\Phi$ with respect to $h$. In local coordinates, $D_{A}$ has coefficient of the form

$$
\left(\begin{array}{ll}
a & 0 \\
0 & d
\end{array}\right)
$$

and $\Phi+\Phi^{\ddagger}$ is of the form

$$
\left(\begin{array}{cc}
0 & b \\
b^{\ddagger} & 0
\end{array}\right) .
$$

Hence $D_{A}$ and $\Phi+\Phi^{\ddagger}$ have coefficients in $\mathfrak{u}(p) \oplus \mathfrak{u}(q)$ and $\mathfrak{p}$, respectively. This implies $D$ is a $\mathrm{U}(p, q)$-Yang-Mills connection.

Denote by $\mathcal{M}_{\text {Dol }}(c)$ the subspace of $\mathbb{C} \mathcal{M}_{\text {Dol }}(c)$ satisfying the hypothesis of Proposition 3.1. Then $\mathcal{M}_{\mathrm{Dol}}(c)$ is homeomorphic to $\mathcal{M}_{D R}(c)$.

The invariants $d_{P}, d_{Q}$ and $\tau$ on $(E, D)$ translate to invariants on the corresponding $\mathrm{U}(p, q)$-Higgs bundles $\left(V_{P} \oplus V_{Q}, \Phi\right)$ :

$$
d_{P}=\operatorname{deg}\left(V_{P}\right), \quad d_{Q}=\operatorname{deg}\left(V_{Q}\right), \quad \tau=2 \frac{q d_{P}-p d_{Q}}{n} .
$$

The subspace of $\mathcal{M}_{\text {Dol }}(c)$ with a fixed Toledo invariant $\tau$ is denoted by $\mathcal{M}_{\text {Dol }}^{\tau}$. 


\section{Group Actions and Kähler Structures on $\mathbb{C} \mathcal{M}_{\text {Dol }}$.}

4.1. The Action of line bundles. The space of holomorphic line bundles, $\mathrm{H}^{1}\left(X, \mathcal{O}^{*}\right)$, acts freely on $\mathbb{C} \mathcal{M}_{\text {Dol }}$ as follows:

$$
\begin{gathered}
\mathrm{H}^{1}\left(X, \mathcal{O}^{*}\right) \times \mathbb{C} \mathcal{M}_{\text {Dol }} \longmapsto \mathbb{C} \mathcal{M}_{\text {Dol }}, \\
(L,(V, \Phi)) \longmapsto(V \otimes L, \Phi \otimes 1),
\end{gathered}
$$

where 1 is the identity map on $L$. An immediate consequence is:

Proposition 4.1. If $c_{1} \equiv c_{2} \bmod n$, then $\mathbb{C} \mathcal{M}_{\text {Dol }}\left(c_{1}\right)$ is homeomorphic to $\mathbb{C} \mathcal{M}_{\text {Dol }}\left(c_{2}\right)$.

4.2. The Action of $\mathrm{H}^{0}(X, \Omega)$. The vector space $\mathrm{H}^{0}(X, \Omega)$ acts freely on $\mathbb{C} \mathcal{M}_{\text {Dol }}$ as follows:

$$
\begin{gathered}
\mathrm{H}^{0}(X, \Omega) \times \mathbb{C} \mathcal{M}_{\text {Dol }} \longmapsto \mathbb{C} \mathcal{M}_{\text {Dol }}, \\
(\phi,(V, \Phi)) \longmapsto(V, \Phi+\phi \mathrm{I}) .
\end{gathered}
$$

The actions of $\mathrm{H}^{1}\left(X, \mathcal{O}^{*}\right)$ and $\mathrm{H}^{0}(X, \Omega)$ commute and the quotient is defined to be

$$
\mathbb{C} \mathcal{N}_{\text {Dol }}=\mathbb{C} \mathcal{M}_{\text {Dol }} /\left(\mathrm{H}^{1}\left(X, \mathcal{O}^{*}\right) \times \mathrm{H}^{0}(X, \Omega)\right) .
$$

The $\mathrm{H}^{1}\left(X, \mathcal{O}^{*}\right)$ action preserves the subspaces $\mathcal{M}_{\text {Dol }}(c)$ and $\mathcal{M}_{\text {Dol }}^{\tau}$. The quotients are defined to be

$$
\begin{aligned}
\mathcal{N}_{\text {Dol }}(c) & =\mathcal{M}_{\text {Dol }}(c) / \mathrm{H}^{1}\left(X, \mathcal{O}^{*}\right), \\
\mathcal{N}_{\text {Dol }}^{\tau} & =\mathcal{M}_{\text {Dol }}^{\tau} / \mathrm{H}^{1}\left(X, \mathcal{O}^{*}\right) .
\end{aligned}
$$

All the spaces constructed so far that contain the symbols $\mathcal{M}_{\text {Dol }}$ or $\mathcal{N}_{\text {Dol }}$ will be loosely referred to as the Dolbeault moduli spaces. The subspace of stable Higgs bundles of a Dolbeault moduli space will be denoted by an $s$ superscript. For example, $\mathbb{C} \mathcal{M}_{\text {Dol }}^{s}$ will denote the subspace of irreducible elements of $\mathbb{C} \mathcal{M}_{\text {Dol }}$.

Remark 1. The Betti, de Rham and Dolbeault moduli spaces $\mathbb{C} \mathcal{M}_{B}$, $\mathbb{C} \mathcal{M}_{\text {Dol }}$ and $\mathbb{C} \mathcal{M}_{\text {Dol }}$ constructed here are variations of those of Simpson's $[22,23,24]$.

With Theorems 2.1 and 2.2, one can obtain the following equivalence relations between the various Betti, de Rham and Dolbeault moduli spaces.

Corollary 4.2. Suppose $\mathcal{M}_{D R}^{\tau} \subset \mathcal{M}_{D R}(c)$. Then one obtains the following commutative diagram:

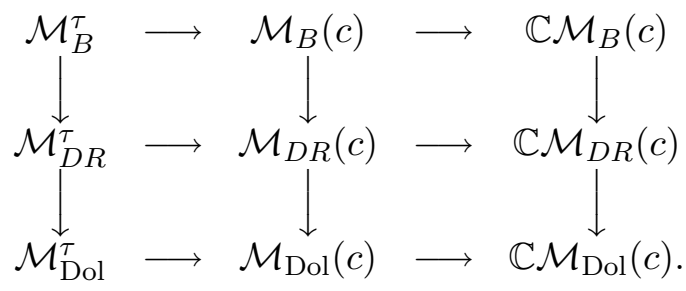


Moreover the horizontal maps are continuous injections and vertical maps are homeomorphisms. One obtains three additional commutative diagrams by respectively replacing the symbol $\mathcal{M}$ by $\mathcal{M}^{s}, \mathcal{N}$ and $\mathcal{N}^{s}$ in the above diagram. In the case of $\mathcal{M}^{s}$, the maps in the commutative diagram are smooth.

4.3. The Dual Higgs Bundles. There is a $\mathbb{Z}_{2}$ action on $\mathbb{C} \mathcal{M}_{\text {Dol }}$. Let $(V, \Phi) \in \mathbb{C} \mathcal{M}_{\text {Dol }}$ where $\Phi$ is a holomorphic map:

$$
\Phi: V \longrightarrow V \otimes \Omega \text {. }
$$

This induces a map on the dual bundles

$$
\Phi^{*}: V^{*} \otimes \Omega^{*} \longrightarrow V^{*} .
$$

Tensoring with $\Omega$,

$$
\Phi^{*} \otimes 1: V^{*} \longrightarrow V^{*} \otimes \Omega,
$$

where 1 denotes the identity map on $\Omega$. This produces the dual Higgs bundle $\left(V^{*}, \Phi^{*} \otimes 1\right)$. We shall abbreviate it as $\left(V^{*}, \Phi^{*}\right)$.

Proposition 4.3. If $(V, \Phi) \in \mathbb{C} \mathcal{M}_{\text {Dol }}(c)$, then $\left(V^{*}, \Phi^{*}\right) \in \mathbb{C} \mathcal{M}_{\text {Dol }}(-c)$.

Proof. One must show that $(V, \Phi)$ is stable (semi-stable) implies $\left(V^{*}, \Phi^{*}\right)$ is stable (semi-stable). Suppose $W_{1} \subset V^{*}$ is $\Phi^{*}$-invariant. Then we have the following commutative diagram

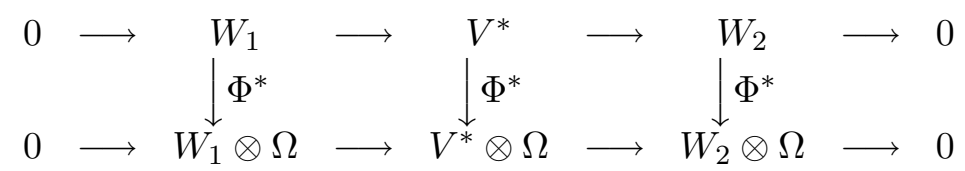

where $W_{2}=V^{*} / W_{1}$. The proposition follows by dualizing the diagram.

In light of Propositions 4.1 and 4.3 we have:

Corollary 4.4. If $c_{2}= \pm c_{1} \bmod n$, then $\mathbb{C} \mathcal{M}_{\text {Dol }}\left(c_{1}\right)$ is homeomorphic to $\mathbb{C} \mathcal{M}_{\text {Dol }}\left(c_{2}\right)$.

4.4. The $U(1)$ and $\mathbb{C}^{*}$-Actions on the Complex Moduli Spaces. If $(V, \Phi) \in \mathbb{C} \mathcal{M}_{\text {Dol }}(c)$, then for $t \in \mathbb{C}^{*},(V, t \Phi) \in \mathbb{C} \mathcal{M}_{\text {Dol }}(c)$. This defines an analytic action $[\mathbf{1 1}, \mathbf{1 2}, \mathbf{2 2}]$

$$
\mathbb{C}^{*} \times \mathbb{C} \mathcal{M}_{\mathrm{Dol}}(c) \longmapsto \mathbb{C} \mathcal{M}_{\mathrm{Dol}}(c) .
$$

Since $\mathrm{U}(1) \subset \mathbb{C}^{*}$, this also induces a $\mathrm{U}(1)$-action on $\mathbb{C} \mathcal{M}_{\text {Dol }}(c)$. 
4.5. The Moment Map. The moduli space $\mathbb{C} \mathcal{M}_{\text {Dol }}(c)^{s}$ is Kähler $[\mathbf{1 1}, \mathbf{1 2}]$. Denote by i, $\omega$ the corresponding complex and symplectic structures, respectively. Define the Morse function $[\mathbf{1 1}, \mathbf{1 2}]$

$$
\begin{gathered}
m: \mathbb{C} \mathcal{M}_{\text {Dol }}(c)^{s} \longrightarrow \mathbb{R}, \\
m(V, \Phi)=2 i \int_{X} \operatorname{tr}\left(\Phi \Phi^{\ddagger}\right),
\end{gathered}
$$

where $\Phi^{\ddagger}$ is the adjoint of $\Phi$ with respect to the Hermitian-Yang-Mills metric on $(E, D)$. Denote by $\mathfrak{X}$ the vector field on $\mathbb{C M}_{\text {Dol }}(c)^{s}$ such that $[\mathbf{1 2}]$

$$
\operatorname{grad} m=\mathrm{i} \mathfrak{X} .
$$

\section{Theorem 4.5.}

1) The map $m$ is proper.

2) The $\mathrm{U}(1)$-action generates $\mathfrak{X}$.

3) The $\mathbb{C}^{*}$ action is analytic with respect to $\mathrm{i}$; therefore, the orbit of $\mathbb{C}^{*}$ is locally an analytic subvariety with respect to i.

Proof. See $[11,12,22]$.

Corollary 4.6. Each component of $\mathbb{C M}_{\mathrm{Dol}}(c)$ contains a point that is a local minimum of $m$.

Corollary 4.7. If the $\mathbb{C}^{*}$ action preserves $\mathcal{M} \subset \mathbb{C M}_{\text {Dol }}(c)^{s}$, then the gradient flow grad $m$ preserves $\mathcal{M}$.

Let $m_{r}$ be the restriction of $m$ to the subspace $\mathcal{M}_{\text {Dol }}^{\tau} \subset \mathbb{C} \mathcal{M}_{\text {Dol }}(c)$.

Corollary 4.8. Every component of $\mathcal{M}_{\mathrm{Dol}}^{\tau}$ contains a point that is a local minimum of $m_{r}$. If $(V, \Phi)$ is stable and is a local minimum of $m_{r}$, then $(V, \Phi)$ is a critical point of $m$.

Proof. Consider

$$
\mathcal{M}_{B}^{\tau} \subset \mathcal{M}_{B}(c) \subset \mathbb{C} \mathcal{M}_{B}(c) .
$$

Since $\mathrm{U}(p, q)$ is closed in $\mathrm{GL}(n, \mathbb{C}), \mathcal{M}_{B}(c)$ is a closed subspace of $\mathbb{C} \mathcal{M}_{B}(c)$. Since the obstruction map $o_{2}$ is continuous, $\mathcal{M}_{B}^{\tau}$ is a closed subspace of $\mathcal{M}_{B}(c)$. Hence $\mathcal{M}_{B}^{\tau}$ is closed in $\mathbb{C} \mathcal{M}_{B}(c)$. Hence by Theorem $4.5, m_{r}$ is proper. Thus each component of $\mathcal{M}_{\text {Dol }}^{\tau}$ contains a local minimum of $m_{r}$.

The points in $\left(\mathcal{M}_{\text {Dol }}^{\tau}\right)^{s}$ are smooth. Suppose $(V, \Phi) \in\left(\mathcal{M}_{\text {Dol }}^{\tau}\right)^{s}$. Then $(V, \Phi)$ is of the form described in Proposition 3.1. Hence the $\mathbb{C}^{*}$ action preserves the subspace $\left(\mathcal{M}_{\text {Dol }}^{\tau}\right)^{s} \subset \mathbb{C} \mathcal{M}_{\text {Dol }}^{s}$. By Corollary 4.7, the gradient flow of $m$ preserves $\left(\mathcal{M}_{\text {Dol }}^{\tau}\right)^{s}$. Hence

$$
\operatorname{grad} m_{r}=\operatorname{grad} m=\mathrm{i} \mathfrak{X} .
$$

If $m_{r}$ is a local minimum at $(V, \Phi)$, then

$$
\operatorname{grad} m(V, \Phi)=\operatorname{grad} m_{r}(V, \Phi)=0 .
$$

Hence $(V, \Phi)$ is a critical point of $m$. 


\section{Bounds on Invariants.}

In this section, we assume $q=1$ and let $n=p+q=p+1$. In light of Proposition 4.3 and Corollary 4.4, one may further assume that $\tau \geq 0$ and $0 \leq c<n$, or equivalently,

$$
s\left(V_{Q}\right) \leq s(V) \leq s\left(V_{P}\right), 0 \leq c<n .
$$

Proposition 5.1. If $(V, \Phi)=\left(V_{P} \oplus V_{Q},\left(\Phi_{1}, \Phi_{2}\right)\right) \in \mathcal{M}_{\text {Dol }}(c)^{s}\left(\mathcal{M}_{\text {Dol }}(c)\right)$, then

$$
\begin{aligned}
& d_{P} \quad<(\leq) \frac{c(n-1)}{n}+(g-1) \\
& d_{Q}>(\geq) \quad \frac{c}{n}-(g-1) .
\end{aligned}
$$

Proof. Suppose $\left(V_{P} \oplus V_{Q}, \Phi\right) \in \mathcal{M}_{\text {Dol }}(c)^{s}$ with $\Phi=\left(\Phi_{1}, \Phi_{2}\right)$ in the notation of Proposition 3.1. Since $s\left(V_{P}\right) \geq s(V)$,

$$
\Phi_{1}: V_{P} \longrightarrow V_{Q} \otimes \Omega
$$

is non-zero.

Construct the canonical factorization for $\Phi_{1}[\mathbf{2 0}]$ : There exist holomorphic bundles $V_{1}, V_{2}$ and $W_{1}, W_{2}$ such that the following diagram

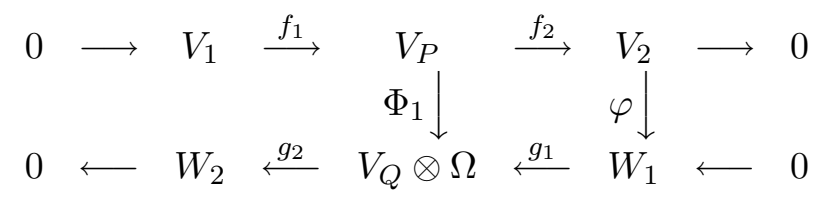

commutes, and the rows are exact, $\operatorname{rank}\left(V_{2}\right)=\operatorname{rank}\left(W_{1}\right)$ and $\varphi$ has full rank at a generic point of $X$. This implies

$$
\left\{\begin{array}{l}
\operatorname{deg}\left(V_{1}\right)+\operatorname{deg}\left(V_{2}\right)=d_{P} \\
\operatorname{deg}\left(W_{1}\right)+\operatorname{deg}\left(W_{2}\right)=d_{Q}+2(g-1) .
\end{array}\right.
$$

Since $\Phi_{1} \not \equiv 0$, we have $\varphi \not \equiv 0, \operatorname{rank}\left(W_{2}\right)=0$ and $W_{1}=V_{Q} \otimes \Omega$.

The case of $p=1$ has been dealt with by Hitchin [11], so we assume $p>1$. Then $V_{1}$ is a $\Phi$-invariant subbundle of positive rank. Stability implies

$$
s\left(V_{1}\right)<s(V)=\left(d_{P}+d_{Q}\right) / n=c / n .
$$

Since the map

$$
V_{2} \stackrel{\varphi}{\longrightarrow} W_{1}=\left(V_{Q} \otimes \Omega\right)
$$

is not trivial,

$$
\operatorname{deg}\left(V_{2}\right) \leq \operatorname{deg}\left(W_{1}\right)=\operatorname{deg}\left(V_{Q} \otimes \Omega\right) .
$$


So one has

This implies

$$
\left\{\begin{array}{l}
s\left(V_{1}\right)<s(V) \\
d_{P}=\operatorname{deg}\left(V_{1}\right)+\operatorname{deg}\left(V_{2}\right) \\
\operatorname{deg}\left(V_{2}\right) \leq d_{Q}+2(g-1) .
\end{array}\right.
$$

$$
d_{P}<\frac{(n-2) c}{n}+d_{Q}+2(g-1)
$$

Since $d_{P}+d_{Q}=c$,

$$
d_{P}<\frac{c(n-1)}{n}+(g-1)
$$

and

$$
d_{Q}>\frac{c}{n}-(g-1)
$$

When $(V, \Phi)$ is semi-stable, one has either $\Phi \not \equiv 0$ or $\Phi \equiv 0$. In the former case, one has $s\left(V_{1}\right) \leq s(V)$ implying

$$
\begin{aligned}
& d_{P} \leq \frac{c(n-1)}{n}+(g-1) \\
& d_{Q} \geq \frac{c}{n}-(g-1) .
\end{aligned}
$$

In the latter case, $V_{p}$ is $\Phi$-invariant. By the assumption $s\left(V_{Q}\right) \leq s\left(V_{P}\right)$, $d_{P}=d_{Q}=0$ and $\tau=0$.

By definition,

$$
\begin{aligned}
\tau & =2 \frac{d_{P}-p d_{Q}}{n} \\
& \leq \frac{2}{n}\left(\frac{c(n-1)}{n}+(g-1)-(n-1) \frac{c}{n}+(n-1)(g-1)\right) \\
& =2(g-1) .
\end{aligned}
$$

Equality holds only when $(V, \Phi)$ is semi-stable but not stable, in which case, the associated flat connection is reducible. This proves Theorem 1.2.

\section{Reducible Higgs Bundles.}

Let $p=2$ and $q=1$ and assume $\tau \geq 0$ and $0 \leq c<3$. By definition, a reducible poly-stable Higgs bundle is a direct sum of stable Higgs bundles of the same slope. These Higgs bundles correspond to the reducible representations in $\mathcal{M}_{B}$. A direct computation shows that if $(V, \Phi)$ is reducible, then

$$
\operatorname{deg}(V)=d_{P}+d_{Q}=0
$$

and the associated Toledo invariant $\tau$ is an even integer. Hence one has: 
Proposition 6.1. If $c=\operatorname{deg}(V) \neq 0$ and $(V, \Phi) \in \mathcal{M}_{\text {Dol }}(c)$, then $(V, \Phi)$ is stable. In particular, $\mathcal{M}_{\mathrm{Dol}}(c)$ is smooth.

An example of a reducible Higgs bundle is $\left(\mathcal{O} \oplus \Omega^{\frac{1}{2}} \oplus \Omega^{-\frac{1}{2}}, \Phi\right)$, where

$$
\Phi: \Omega^{\frac{1}{2}} \longrightarrow \Omega^{-\frac{1}{2}} \otimes \Omega
$$

is a holomorphic bundle isomorphism. That is, $\Phi$ is of the form

$$
\left(\begin{array}{lll}
0 & 0 & 0 \\
0 & 0 & 0 \\
0 & 1 & 0
\end{array}\right) .
$$

The Toledo invariant in this case is $2(g-1)$. All the flat $\mathrm{U}(2,1)$-connections with $\tau=2(g-1)$ are reducible by Proposition 5.1. The fact that there is no irreducible deformation for the $\mathrm{U}(2,1)$-connections with $\tau=2(g-1)$ was first demonstrated by Toledo [26]. In particular, this component is connected $[6,11]$.

\section{Hodge Bundles and Deformation.}

Let $p=2$ and $q=1$ and assume $\tau \geq 0$ and $0 \leq c<3$. A Hodge bundle on $X$ is a direct sum of holomorphic bundles [22]

$$
V=\bigoplus_{s, t} V^{s, t}
$$

together with holomorphic maps (Higgs field)

$$
\Phi_{i}: V^{s, t} \longrightarrow V^{s-1, t+1} \otimes \Omega \text {. }
$$

An immediate consequence of Proposition 3.1 is:

Corollary 7.1. Suppose $\left(V_{P} \oplus V_{Q},\left(\Phi_{1}, \Phi_{2}\right)\right) \in \mathcal{M}_{\text {Dol }}(c)$ (in the notations of Proposition 3.1). Then $\left(V_{P} \oplus V_{Q},\left(\Phi_{1}, \Phi_{2}\right)\right)$ is a Hodge bundle if and only if $\left(V_{P} \oplus V_{Q},\left(\Phi_{1}, \Phi_{2}\right)\right)$ is either binary or ternary in the following sense:

1) Binary: $\Phi_{2} \equiv 0$.

2) Ternary: $V_{P}=V_{1} \oplus V_{2}$ and the Higgs field consists of two maps:

$$
\begin{aligned}
& \Phi_{1}: V_{2} \longrightarrow V_{Q} \otimes \Omega, \\
& \Phi_{2}: V_{Q} \longrightarrow V_{1} \otimes \Omega .
\end{aligned}
$$

Denote by $B\left(d_{P}, d_{Q}\right)$ the space of all poly-stable (or $S$-equivalence classes of semi-stable) binary Hodge bundles $\left(V_{P} \oplus V_{Q},\left(\Phi_{1}, 0\right)\right)$ with $\operatorname{deg}\left(V_{P}\right)=d_{P}$ and $\operatorname{deg}\left(V_{Q}\right)=d_{Q}$. Denote by $T\left(d_{1}, d_{2}, d_{Q}\right)$ the space of all poly-stable (or $S$-equivalence classes of semi-stable) ternary Hodge bundles $\left(V_{1} \oplus V_{2} \oplus\right.$ $\left.V_{Q},\left(\Phi_{1}, \Phi_{2}\right)\right)$ with $\operatorname{deg}\left(V_{1}\right)=d_{1}, \operatorname{deg}\left(V_{2}\right)=d_{2}$ and $\operatorname{deg}\left(V_{Q}\right)=d_{Q}$. Denote the subspaces of stable Hodge bundles by $B\left(d_{P}, d_{Q}\right)^{s}, T\left(d_{1}, d_{2}, d_{Q}\right)^{s}$. When $\tau$ is not an integer, these are the type $(2,1)$ and $(1,1,1)$ spaces in [8]. Note the $(1,2)$ types give $\tau<0$ and therefore need not be considered here. 
Proposition 7.2. Every stable binary Hodge bundle in $\left(\mathcal{M}_{\text {Dol }}^{\tau}\right)^{s}$ may be deformed to a stable ternary Hodge bundle within $\mathcal{M}_{\text {Dol }}^{\tau}$.

A family (or flat family) of Higgs pairs $\left(V_{Y}, \Phi_{Y}\right)$ is a variety $\mathrm{Y}$ such that there is a vector bundle $V_{Y}$ on $X \times Y$ together with a section $\Phi_{Y} \in$ $\Gamma\left(Y,\left(\pi_{Y}\right)_{*}\left(\pi_{X}^{*} \Omega \otimes \operatorname{End}\left(V_{Y}\right)\right)\right)[\mathbf{1 8}]$. $\mathbb{C} \mathcal{M}_{\text {Dol }}$ being a moduli space implies that if $Y$ is a family of stable (poly-stable or $S$-equivalence classes of semi-stable) Higgs bundles, then there is a natural morphism $[\mathbf{1 5}, \mathbf{1 7}]$

$$
t: Y \longrightarrow \mathbb{C} \mathcal{M}_{\text {Dol }} \text {. }
$$

Moreover $t$ takes every point $y \in Y$ to the point of $\mathbb{C} \mathcal{M}_{\text {Dol }}$ that corresponds to the Higgs bundle in the family over $y[\mathbf{1 5}, \mathbf{1 7}, \mathbf{1 8}]$.

The space $\mathcal{M}_{\text {Dol }}(c)$ is a subvariety of $\mathbb{C} \mathcal{M}_{\text {Dol }}(c)$; hence, to show that two stable (poly-stable or $S$-equivalence classes of semi-stable) Higgs bundles $\left(V_{1}, \Phi_{1}\right)$ and $\left(V_{2}, \Phi_{2}\right)$ belong to the same component of $\mathcal{M}_{\text {Dol }}(c)$, it suffices to exhibit a connected family $Y$ (within $\mathcal{M}_{\text {Dol }}(c)$ ) of stable (poly-stable or $S$-equivalence classes of semi-stable) Higgs bundles containing both $\left(V_{1}, \Phi_{1}\right)$ and $\left(V_{2}, \Phi_{2}\right)$.

Proof. Suppose $(V, \Phi)=\left(V_{P} \oplus V_{Q},\left(\Phi_{1}, 0\right)\right) \in B\left(d_{P}, d_{Q}\right)^{s} \subset\left(\mathcal{M}_{\mathrm{Dol}}^{\tau}\right)^{s}$. Since $s\left(V_{P}\right) \geq s(V)$ (This is due to the assumption $\tau \geq 0$, and $0 \leq c<3$ ), $\Phi_{1} \not \equiv 0$. Construct the canonical factorization for $\Phi_{1}$ :

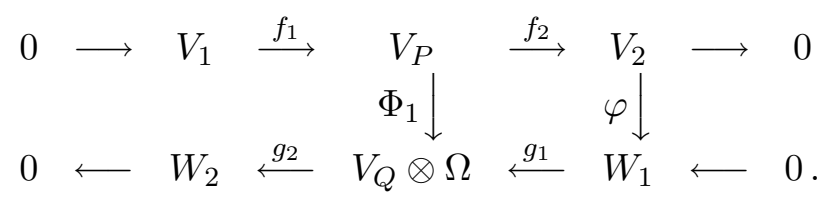

$V_{1}$ being $\Phi_{1}$ invariant implies

$$
\operatorname{deg}\left(V_{1}\right)=s\left(V_{1}\right)<s(V) \leq s\left(V_{P}\right) \leq s\left(V_{2}\right)=\operatorname{deg}\left(V_{2}\right) .
$$

The space $\operatorname{Pic}^{0}(X)$ of line bundles of degree 0 over $X$ is identified with the Jacobi variety $J_{0}(X)$. Construct the universal bundle $[2,19]$

$$
U \longrightarrow X \times J_{0}(X)
$$

such that $U$ restricts to the bundle $L \otimes V_{1} \otimes V_{2}^{-1}$ on $(X, L)$. Let $\pi$ be the projection

$$
\pi: X \times J_{0}(X) \longrightarrow J_{0}(X) .
$$

Applying the right derived functor $R^{1}$ to $\pi$ gives the sheaf $\mathcal{F}=R^{1} \pi_{*}(U)$ [10] such that

$$
\left.\mathcal{F}\right|_{L}=\mathrm{H}^{1}\left(X, L \otimes V_{1} \otimes V_{2}^{-1}\right) .
$$

Since

$$
\operatorname{deg}\left(L \otimes V_{1} \otimes V_{2}^{-1}\right)=\operatorname{deg}\left(V_{1}\right)-\operatorname{deg}\left(V_{2}\right)<0,
$$


by Riemann-Roch,

$$
\begin{aligned}
h^{1}\left(L \otimes V_{1} \otimes V_{2}^{-1}\right) & =h^{0}\left(L \otimes V_{1} \otimes V_{2}^{-1}\right)-\operatorname{deg}\left(L \otimes V_{1} \otimes V_{2}^{-1}\right)+(g-1) \\
& =\operatorname{deg}\left(V_{2}\right)-\operatorname{deg}\left(V_{1}\right)+(g-1)
\end{aligned}
$$

is a constant. By Grauert's theorem, $\mathcal{F}$ is locally free, hence, is associated with a vector bundle

$$
F \longmapsto J_{0}(X)
$$

of rank $\operatorname{deg}\left(V_{2}\right)-\operatorname{deg}\left(V_{1}\right)+(g-1)$. In particular the total space $F$ is smooth and parameterizes extensions $[\mathbf{9}, \mathbf{1 0}]$ :

$$
0 \longrightarrow L \otimes V_{1} \stackrel{f_{3}}{\longrightarrow} W_{P} \stackrel{f_{4}}{\longrightarrow} V_{2} \longrightarrow 0
$$

for fixed $V_{1}, V_{2}$. Tensoring the above sequence with $\Omega$ gives:

$$
0 \longrightarrow L \otimes V_{1} \otimes \Omega \stackrel{g_{3}}{\longrightarrow} W_{P} \otimes \Omega \stackrel{g_{4}}{\longrightarrow} V_{2} \otimes \Omega \longrightarrow 0 .
$$

Fix $\varphi$. Then $F$ also parameterizes a family of Higgs bundles $\left(W_{P}, \Phi_{1}^{\prime}\right)$ that fit into the factorization

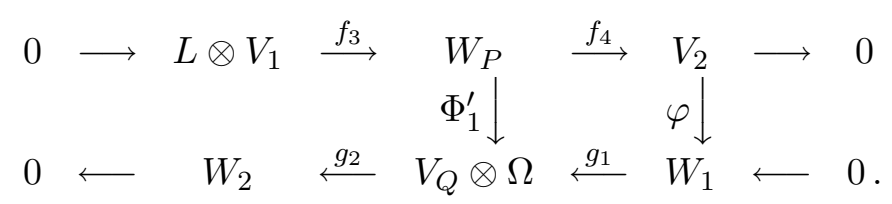

Let $\mathcal{V} \subset F$ be the subset of stable extensions (i.e., $W_{P} \in \mathcal{V}$ implies $W_{P}$ is a stable holomorphic bundle [19]).

Lemma 7.3. $\mathcal{V} \cap \mathrm{H}^{1}\left(L \otimes V_{1} \otimes V_{2}^{-1}\right)$ and $\mathcal{V}$ are open and dense in $\mathrm{H}^{1}(L \otimes$ $\left.V_{1} \otimes V_{2}^{-1}\right)$ and $F$, respectively. Moreover if $W_{P} \in \mathcal{V}$, then $\left(W_{P} \oplus V_{Q},\left(\Phi_{1}^{\prime}, 0\right)\right)$ is stable.

Proof. Since $\operatorname{deg}\left(L \otimes V_{1}\right)<\operatorname{deg}\left(V_{2}\right)$ for each $L \in J_{0}(X)$, by a theorem of Lange and Narasimhan [13], there always exists a stable extension $W_{P} \in$ $\mathrm{H}^{1}\left(L \otimes V_{1} \otimes V_{2}^{-1}\right)$. In addition, a theorem of Maruyama states that being stable is an open property [14]. The open dense property follows from the smoothness of $F$ and $\mathrm{H}^{1}\left(L \otimes V_{1} \otimes V_{2}^{-1}\right)$.

Let $p_{P}, p_{Q}$ be the projections of $W_{P} \oplus V_{Q}$ onto its $W_{P}$ and $V_{Q}$ factors, respectively. Suppose $W$ is $\left(\Phi_{1}^{\prime}, 0\right)$-invariant. Suppose $W$ has rank 1. If $P_{Q}(W)=0$, then $W=L \otimes V_{1}$; otherwise, $\operatorname{deg}(W) \leq \operatorname{deg}\left(V_{Q}\right)$. In either case, $s(W)<s(V)$. Suppose $W$ has rank 2. If $p_{Q}(W)=0$, then $W=W_{P}$ and $s(W)<s(V)$. Suppose $P_{Q}(W) \neq 0$. Then there exists a line bundle $L_{1}$ such that

$$
0 \longrightarrow L_{1} \longrightarrow W \stackrel{p_{Q}}{\longrightarrow} p_{Q}(W) \longrightarrow 0 .
$$

Now let $L_{P}=p_{P}\left(L_{1}\right) \subset W_{P}$. Then

$$
\operatorname{deg}(W)=\operatorname{deg}\left(L_{1}\right)+\operatorname{deg}\left(p_{Q}(W)\right) \leq \operatorname{deg}\left(L_{P}\right)+\operatorname{deg}\left(V_{Q}\right) .
$$


Since $W_{P}$ is stable, $s\left(L_{P}\right)<s\left(W_{P}\right)$. By the assumptions $\tau \geq 0$ and $0 \leq c<$ 3 , one has $s\left(V_{Q}\right) \leq 0$ and $s\left(W_{P}\right) \geq 0$. Therefore,

$$
\begin{gathered}
s(W) \leq s\left(L_{P} \oplus V_{Q}\right)=\frac{s\left(L_{P}\right)+s\left(V_{Q}\right)}{2}<\frac{s\left(W_{P}\right)+s\left(V_{Q}\right)}{2} \\
=\frac{\operatorname{deg}\left(W_{P}\right)}{4}+\frac{\operatorname{deg}\left(V_{Q}\right)}{2} \leq \frac{\operatorname{deg}\left(W_{P}\right)+\operatorname{deg}\left(V_{Q}\right)}{3}=s(V) .
\end{gathered}
$$

Thus $\left(W_{P} \oplus V_{Q},\left(\Phi_{1}^{\prime}, 0\right)\right)$ is stable.

Since $\Phi_{1} \not \equiv 0, \operatorname{deg}\left(V_{2}\right) \leq d_{Q}+2(g-1)$ and

$$
\operatorname{deg}\left(V_{1}\right)=d_{P}-\operatorname{deg}\left(V_{2}\right) \geq d_{P}-d_{Q}-2(g-1) .
$$

Hence

$$
\operatorname{deg}\left(V_{Q}^{-1} \otimes V_{1} \otimes \Omega\right)=-d_{Q}+\operatorname{deg}\left(V_{1}\right)+2(g-1) \geq d_{P}-2 d_{Q}>0 .
$$

Hence there exists $L^{\prime} \in J(X)$ such that

$$
h^{0}\left(V_{Q}^{-1} \otimes L^{\prime} \otimes V_{1} \otimes \Omega\right)>0
$$

implying there exists a non-trivial holomorphic map

$$
\phi: V_{Q} \longrightarrow L^{\prime} \otimes V_{1} \otimes \Omega \text {. }
$$

Fix $\phi \not \equiv 0$. By Lemma 7.3, the family parameterized by $\mathcal{V}$ contains both $\left(V_{P} \oplus V_{Q},\left(\Phi_{1}, 0\right)\right)$ and $\left(W_{P} \oplus V_{Q},\left(\Phi_{1}^{\prime}, 0\right)\right)$ implying there is deformation between the two.

Set $L=L^{\prime}$ and $\Phi_{2}^{\prime}=g_{3} \circ \phi$. Then the family of stable Higgs bundles parameterized by $\mathrm{H}^{0}\left(X, V_{Q}^{-1} \otimes L^{\prime} \otimes V_{1} \otimes \Omega\right)$ contains $\left(W_{P} \oplus V_{Q},\left(\Phi_{1}^{\prime}, 0\right)\right)$ and $\left(W_{P} \oplus V_{Q},\left(\Phi_{1}^{\prime}, \Phi_{2}^{\prime}\right)\right)$.

Now the family of bundle extensions of $V_{2}$ by $L^{\prime} \otimes V_{1}$ is $\mathrm{H}^{1}\left(L^{\prime} \otimes V_{1} \otimes V_{2}^{-1}\right)$. With a fixed $\phi$ and the canonical factorization with $\varphi$ fixed, $\mathrm{H}^{1}\left(L^{\prime} \otimes V_{1} \otimes\right.$ $\left.V_{2}^{-1}\right)$ parameterizes a family of Higgs bundles. This family contains $\left(W_{P} \oplus\right.$ $\left.V_{Q},\left(\Phi_{1}^{\prime}, \Phi_{2}^{\prime}\right)\right)$. The zero element in $\mathrm{H}^{1}\left(L^{\prime} \otimes V_{1} \otimes V_{2}^{-1}\right)$ corresponds to the bundle extension

$$
0 \longrightarrow L^{\prime} \otimes V_{1} \stackrel{f_{5}}{\longrightarrow}\left(L^{\prime} \otimes V_{1}\right) \oplus V_{2} \stackrel{f_{6}}{\longrightarrow} V_{2} \longrightarrow 0 .
$$

Tensoring with $\Omega$ gives

$$
0 \longrightarrow L^{\prime} \otimes V_{1} \otimes \Omega \stackrel{g_{5}}{\longrightarrow}\left(\left(L^{\prime} \otimes V_{1}\right) \oplus V_{2}\right) \otimes \Omega \stackrel{g_{6}}{\longrightarrow} V_{2} \otimes \Omega \longrightarrow 0 .
$$

Lemma 7.4. If $\left(W_{P} \oplus V_{Q},\left(\Phi_{1}^{\prime}, \Phi_{2}^{\prime}\right)\right)$ is stable (semi-stable), then $\mathrm{H}^{1}\left(L^{\prime} \otimes\right.$ $\left.V_{1} \otimes V_{2}^{-1}\right)$ parameterizes a stable (semi-stable) family.

Proof. Suppose $\left(U_{p} \oplus V_{Q},\left(\Psi_{1}, \Psi_{2}\right)\right) \in \mathrm{H}^{1}\left(L^{\prime} \otimes V_{1} \otimes V_{2}^{-1}\right)$ and $W \subset U_{P} \oplus V_{Q}$ is $\left(\Psi_{1}, \Psi_{2}\right)$-invariant. Since $\varphi, \phi \not \equiv 0$, one has $W=V_{1}$ or $W=V_{Q} \oplus V_{1}$. A direct computation shows $s(W)<s\left(U_{P} \oplus V_{Q}\right)\left(s(W) \leq s\left(U_{P} \oplus V_{Q}\right)\right)$. 
Proposition 7.2 follows from Lemma 7.4 because the family of Higgs bundles parameterized by $\mathrm{H}^{1}\left(L^{\prime} \otimes V_{1} \otimes V_{2}^{-1}\right)$ contains $\left(W_{P} \oplus V_{Q},\left(\Phi_{1}^{\prime}, \Phi_{2}^{\prime}\right)\right)$ and $\left(\left(L^{\prime} \otimes V_{1}\right) \oplus V_{2} \oplus V_{Q},\left(g_{1} \circ \varphi \circ f_{6}, g_{5} \circ \phi\right)\right)$.

To summarize, a stable binary Hodge bundle $\left(V_{P} \oplus V_{Q},\left(\Phi_{1}, 0\right)\right)$ is first deformed to $\left(W_{P} \oplus V_{Q},\left(\Phi_{1}^{\prime}, 0\right)\right)$ such that non-trivial holomorphic maps exist between $V_{Q}$ and $\left(L^{\prime} \otimes V_{1}\right) \otimes \Omega \subset W_{P} \otimes \Omega$. Such a non-trivial map $\Phi_{2}^{\prime}$ is then chosen and attached to the existing Higgs field $\Phi_{1}^{\prime}$. Finally $W_{P}$ is deformed to a direct sum making the resulting stable Higgs bundle a ternary Hodge bundle.

Let $B=B(0,0) \backslash\left(B(0,0)^{s} \cup T(0,0,0)\right)$.

Proposition 7.5. $B$ is connected and can be deformed to a stable ternary Hodge bundle in $\mathcal{M}_{\text {Dol }}^{0}$.

Proof. Consider the space $U \times J_{0}(X)$, where $J_{0}(X)$ is the Jacobi variety identified with the set of holomorphic line bundles of degree zero on $X$ and $U$ is the moduli space of rank-2 poly-stable holomorphic bundles of degree 0 on $X$. The space $U$ is connected $[\mathbf{2}, \mathbf{1 9}]$. Hence $U \times J_{0}(X)$ is connected. Each poly-stable Higgs bundle in $B$ is contained in the family of Higgs bundles parameterized by $U \times J_{0}(X)$. Hence the natural morphism

$$
t: U \times J_{0}(X) \longrightarrow B
$$

is surjective. This proves that the set $B$ is connected.

Choose holomorphic line bundles $V_{1}, V_{2}, V_{Q}$ of degrees $-1,1,0$, respectively such that

Choose

$$
\begin{gathered}
h^{0}\left(X, V_{2}^{-1} \otimes V_{Q} \otimes \Omega\right)>0, \\
h^{0}\left(X, V_{Q}^{-1} \otimes V_{1} \otimes \Omega\right)>0 .
\end{gathered}
$$

$$
\begin{aligned}
& 0 \not \equiv \psi_{1} \in \mathrm{H}^{0}\left(X, V_{2}^{-1} \otimes V_{Q} \otimes \Omega\right) \\
& 0 \not \equiv \psi_{2} \in \mathrm{H}^{0}\left(X, V_{Q}^{-1} \otimes V_{1} \otimes \Omega\right) .
\end{aligned}
$$

The space of extension of $V_{2}$ by $V_{1}$,

$$
0 \longrightarrow V_{1} \stackrel{f_{1}}{\longrightarrow} V_{P} \stackrel{f_{2}}{\longrightarrow} V_{2} \longrightarrow 0,
$$

is $\mathrm{H}^{1}\left(X, V_{1} \otimes V_{2}^{-1}\right)$. Tensoring the exact sequence with $\Omega$ gives

$$
0 \longrightarrow V_{1} \otimes \Omega \stackrel{g_{1}}{\longrightarrow} V_{P} \otimes \Omega \stackrel{g_{2}}{\longrightarrow} V_{2} \otimes \Omega \longrightarrow 0 .
$$

Since $\operatorname{deg}\left(V_{1}\right)<\operatorname{deg}\left(V_{2}\right)$, by the theorem of Lange and Narasimhan [13], stable extensions always exist. Fix a stable extension $V_{P}$ and set

$$
\begin{aligned}
& \Phi_{1}=\psi_{1} \circ f_{2}, \\
& \Phi_{2}=g_{1} \circ \psi_{2} .
\end{aligned}
$$

Note $\left(V_{P} \oplus V_{Q}, 0\right) \in B$. The connected family

$$
F C=\mathrm{H}^{0}\left(X, V_{2}^{-1} \otimes V_{Q} \otimes \Omega\right) \times \mathrm{H}^{0}\left(X, V_{Q}^{-1} \otimes V_{1} \otimes \Omega\right)
$$


of Higgs bundles contains $\left(V_{P} \oplus V_{Q}, 0\right)$ and $\left(V_{P} \oplus V_{Q},\left(\Phi_{1}, \Phi_{2}\right)\right)$. Note the family $F C$ contains semi-stable Higgs bundles. This is allowed since the points in the moduli space $\mathcal{M}_{\text {Dol }}$ are also interpreted as $S$-equivalence classes of semi-stable Higgs bundles. However one may choose $F C$ to be a strictly poly-stable family:

$$
\begin{aligned}
F C= & \left(\mathrm{H}^{0}\left(X, V_{2}^{-1} \otimes V_{Q} \otimes \Omega\right) \times \mathrm{H}^{0}\left(X, V_{Q}^{-1} \otimes V_{1} \otimes \Omega\right)\right) \backslash \\
& \left(\left(\{0\} \times \mathrm{H}^{0}\left(X, V_{Q}^{-1} \otimes V_{1} \otimes \Omega\right)\right) \cup\left(\mathrm{H}^{0}\left(X, V_{2}^{-1} \otimes V_{Q} \otimes \Omega\right) \times\{0\}\right)\right) .
\end{aligned}
$$

Since $V_{P}$ is stable, by Lemma 7.3, any element in $F C$ is semi-stable. Hence the family $F C$ provides a deformation between $\left(V_{P} \oplus V_{Q}, 0\right)$ and $\left(V_{P} \oplus\right.$ $\left.V_{Q},\left(\Phi_{1}, \Phi_{2}\right)\right)$. The cohomology $\mathrm{H}^{1}\left(X, V_{1} \otimes V_{2}^{-1}\right)$ parameterizes bundle extensions of $V_{2}$ by $V_{1}$ and also parameterizes a family of Higgs bundles with fixed $\psi_{1}, \psi_{2}$. By Lemma 7.4 , this is a stable family which contains $\left(V_{P} \oplus V_{Q},\left(\Phi_{1}, \Phi_{2}\right)\right)$ and $\left(V_{1} \oplus V_{2} \oplus V_{Q},\left(\psi_{1} \circ f_{4}, g_{3} \circ \psi_{2}\right)\right)$ where $f_{3}, f_{4}, g_{3}, g_{4}$ come from the trivial extensions

$$
\begin{gathered}
0 \longrightarrow V_{1} \stackrel{f_{3}}{\longrightarrow} V_{1} \oplus V_{2} \stackrel{f_{4}}{\longrightarrow} V_{2} \longrightarrow 0, \\
0 \longrightarrow V_{1} \otimes \Omega \stackrel{g_{3}}{\longrightarrow}\left(V_{1} \oplus V_{2}\right) \otimes \Omega \stackrel{g_{4}}{\longrightarrow} V_{2} \otimes \Omega \longrightarrow 0 .
\end{gathered}
$$

Hence $\mathrm{H}^{1}\left(X, V_{1} \otimes V_{2}^{-1}\right)$ provides a deformation between $\left(V_{P} \oplus V_{Q},\left(\Phi_{1}, \Phi_{2}\right)\right)$ and $\left(V_{1} \oplus V_{2} \oplus V_{Q},\left(\psi_{1} \circ f_{4}, g_{3} \circ \psi_{2}\right)\right) \in T(-1,1,0)$.

To summarize, one first shows that the space $B$ is connected. Then choose a specific element $\left(V_{P} \oplus V_{Q}, 0\right) \in B$ with $V_{P}$ a stable extension of $V_{2}$ by $V_{1}$ and that there exists non-trivial holomorphic maps

$$
\begin{array}{ll}
\psi_{1}: & V_{2} \longrightarrow V_{Q} \otimes \Omega \\
\psi_{2}: & V_{Q} \longrightarrow V_{1} \otimes \Omega .
\end{array}
$$

This provides a deformation from $\left(V_{P} \oplus V_{Q}, 0\right)$ to $\left(V_{P} \oplus V_{Q},\left(\Phi_{1}, \Phi_{2}\right)\right)$. Finally, since $V_{P}$ is an extension of $V_{2}$ by $V_{1},\left(V_{P} \oplus V_{Q},\left(\Phi_{1}, \Phi_{2}\right)\right)$ is deformed to $\left(V_{1} \oplus V_{2} \oplus V_{Q},\left(\psi_{1} \circ f_{4}, g_{3} \circ \psi_{2}\right)\right)$ in $\mathrm{H}^{1}\left(X, V_{1} \otimes V_{2}^{-1}\right)$.

Corollary 7.6. Every Binary Hodge bundle can be deformed to a ternary Hodge bundle.

Proof. Every poly-stable reducible Hodge bundle is either ternary or in $B$. The result then follows from Proposition 7.2 and 7.5.

Lemma 7.7. For fixed integers $d_{1}, d_{2}, d_{3}, T\left(d_{1}, d_{2}, d_{3}\right)$ is connected.

Proof. We first consider the stable bundles. Stability implies the Higgs fields $\Phi_{1}, \Phi_{2}$ are not identically zero. Denote by $J_{d}(X)$ the Jacobi variety identified with the set of holomorphic line bundles of degree $d$. For each $L_{1} \in J_{d_{1}}(X)$, the set of all $\left(L_{3}, \Phi_{2}\right)$ such that $L_{3} \in J_{d_{3}}(X)$ and

$$
0 \not \equiv \Phi_{2} \in \mathrm{H}^{0}\left(X, L_{3}^{-1} \otimes L_{1} \otimes \Omega\right)
$$


is $\mathbb{C}^{*} \times S y m^{d_{1}+2(g-1)-d_{3}} X$, where $S y m^{d} X$ is the $d$-th symmetric product of $X$. Hence the set of all triples $\left(L_{3}, L_{1}, \Phi_{2}\right)$ such that

$$
L_{3} \stackrel{\Phi_{2}}{\longmapsto} L_{1} \otimes \Omega
$$

with $\Phi_{2} \not \equiv 0$ is the space $\left(\mathbb{C}^{*} \times S y m^{d_{1}+2(g-1)-d_{3}} X\right) \times J_{d_{1}}(X)$.

Similarly, for each $L_{3} \in J_{d_{3}}(X)$, the space of all triples $\left(L_{2}, L_{3}, \Phi_{1}\right)$ such that

$$
L_{2} \stackrel{\Phi_{1}}{\longmapsto} L_{3} \otimes \Omega
$$

with $\Phi_{1} \not \equiv 0$ is $\mathbb{C}^{*} \times S y m^{d_{3}+2(g-1)-d_{2}} X$. The set of Higgs bundles parameterized by the total space

$$
S=\left(\mathbb{C}^{*} \times S y m^{d_{3}+2(g-1)-d_{2}} X\right) \times\left(\mathbb{C}^{*} \times S y m^{d_{1}+2(g-1)-d_{3}} X\right) \times J_{d_{3}}(X)
$$

contains every Higgs bundle in $T\left(d_{1}, d_{2}, d_{3}\right)$. Hence the natural morphism

$$
t: S \longrightarrow T\left(d_{1}, d_{2}, d_{3}\right)
$$

is surjective. Since $S$ is connected, $T\left(d_{1}, d_{2}, d_{3}\right)$ is connected.

The reducible bundles consist of $T(0,0,0)$ and $T\left(0, d_{2},-d_{2}\right)$. All polystable Higgs bundles associated with the points in $T(0,0,0)$ and $T\left(0, d_{2},-d_{2}\right)$ are contained in the families parameterized by

$$
S_{1}=J_{0}(X) \times J_{0}(X) \times J_{0}(X)
$$

and

$$
S_{2}=\left(\mathbb{C}^{*} \times S y m^{2(g-1)-2 d_{2}} X\right) \times J_{-d_{2}}(X) \times J_{0}(X),
$$

respectively. Both $S_{1}, S_{2}$ are connected. Since the natural morphisms

$$
\begin{gathered}
t_{1}: S_{1} \longrightarrow T(0,0,0) \\
t_{2}: S_{2} \longrightarrow T\left(0, d_{2},-d_{2}\right)
\end{gathered}
$$

are surjective, both $T(0,0,0)$ and $T\left(0, d_{2},-d_{2}\right)$ are connected.

Proposition 7.8. Every component of $\mathcal{M}_{\text {Dol }}^{\tau}$ contains a Hodge bundle.

Proof. By Corollary 4.8, every component of $\mathcal{M}_{\text {Dol }}^{\tau}$ contains a local minimum $(V, \Phi)$ of $m_{r}$. If $(V, \Phi)$ is a smooth point, then $(V, \Phi)$ is a critical point of $m$. A theorem of Hitchin and Simpson implies that $(V, \Phi)$ is a Hodge bundle $[\mathbf{1 2}, \mathbf{2 2}]$. Singular points of $\mathcal{M}_{\text {Dol }}^{\tau}$ correspond to reducible Higgs bundles. The space of all reducible Higgs bundles correspond to either the space of $\mathrm{U}(2) \times \mathrm{U}(1)$ representations or the space of $\mathrm{U}(1) \times \mathrm{U}(1,1)$ representations. Each component of $\mathrm{U}(2) \times \mathrm{U}(1)$ and $\mathrm{U}(1) \times \mathrm{U}(1,1)$ representations contains points that correspond to Hodge bundles [11]. In fact, these points are exactly the ones corresponding to the points in $B$ and $T\left(0, d_{2},-d_{2}\right)$.

Let $K$ be a divisor of $\Omega$ and let

$$
w: X \longrightarrow|K| \cong \mathbb{C P}^{g-1}
$$

be the canonical map [10]. 
Lemma 7.9. $\Omega$ has a section with simple zeros.

Proof. The linear system $|K|$ is base point free [10]. If $X$ is hyperelliptic, then the map $w$ is a $2-1$ branch map into $\mathbb{C P}^{g-1}$ and an embedding otherwise. In both cases, by Bertini's theorem, there exists a hyperplane $\mathrm{H} \in \mathbb{C P}^{g-1}$ such that $\mathrm{H} \cap X$ is regular. Then $w^{-1}(\mathrm{H})$ is an effective divisor equivalent to $K$ and with simple zeros.

Choose

$$
K=\left\{x_{1}, x_{2}, \ldots, x_{2(g-1)}\right\},
$$

such that the $x_{i}$ 's are all distinct.

Proposition 7.10. Let $0 \leq \tau<2(g-1)$. Suppose

$$
T\left(d_{1}-1, d_{2}+1, d_{Q}\right), T\left(d_{1}, d_{2}, d_{Q}\right) \subset \mathcal{M}_{\text {Dol }}^{\tau} .
$$

Then there is deformation between $T\left(d_{1}, d_{2}, d_{Q}\right)$ and $T\left(d_{1}-1, d_{2}+1, d_{Q}\right)$ within $\mathcal{M}_{\mathrm{Dol}}^{\tau}$.

Proof. Suppose

$$
\begin{gathered}
\left(V_{1} \oplus V_{2} \oplus V_{Q},\left(\Phi_{1}, \Phi_{2}\right)\right) \in T\left(d_{1}-1, d_{2}+1, d_{Q}\right), \\
\left(U_{1} \oplus U_{2} \oplus U_{Q},\left(\Psi_{1}, \Psi_{2}\right)\right) \in T\left(d_{1}, d_{2}, d_{Q}\right) .
\end{gathered}
$$

By the semi-stability of $\left(U_{1} \oplus U_{2} \oplus U_{Q},\left(\Psi_{1}, \Psi_{2}\right)\right)$ and the assumptions $\tau \geq$ $0,0 \leq c<3$, one has $d_{Q} \leq 0$ and

$$
d_{1}-1<d_{1} \leq \frac{d_{P}+d_{Q}}{3}<1
$$

hence,

$$
d_{1}-1<d_{1} \leq 0 \text { and } d_{2}+1>0 .
$$

This implies $\left(V_{1} \oplus V_{2} \oplus V_{Q},\left(\Phi_{1}, \Phi_{2}\right)\right)$ is stable. Hence $\Phi_{1} \not \equiv 0$ and

$$
-\operatorname{deg}\left(V_{2}\right)+d_{Q}+2(g-1) \geq 0 \text {. }
$$

On the other hand, $\operatorname{deg}\left(V_{1}\right)+\operatorname{deg}\left(V_{2}\right)=d_{P}$, so

$$
\begin{gathered}
d_{P}-\operatorname{deg}\left(V_{1}\right)-d_{Q} \leq 2(g-1), \\
-d_{1}<1-d_{1}=-\operatorname{deg}\left(V_{1}\right) \leq-d_{P}+d_{Q}+2(g-1) \leq 2(g-1) .
\end{gathered}
$$

In light of Lemma 7.7, it suffices to demonstrate the existence of $\left(U_{1} \oplus\right.$ $\left.U_{2} \oplus U_{Q},\left(\Psi_{1}, \Psi_{2}\right)\right) \in T\left(d_{1}, d_{2}, d_{Q}\right)$ and $\left(V_{1} \oplus V_{2} \oplus U_{Q},\left(\Phi_{1}, \Phi_{2}\right)\right) \in T\left(d_{1}-\right.$ $\left.1, d_{2}+1, d_{Q}\right)$ and a deformation between the two.

Since $|K|$ is base point free, there exists $K^{\prime} \in|K|$ such that

$$
K^{\prime}=\left\{y_{1}, y_{2}, \ldots, y_{2(g-1)}\right\}
$$


with $y_{i} \neq x_{2(g-1)}$ for all $1 \leq i \leq 2 g$. The bounds on the degrees of the various bundles allow us to construct the following divisors:

$$
\left\{\begin{aligned}
D_{1} & =\left\{-x_{1}, \ldots,-x_{-\operatorname{deg}\left(U_{1}\right)}\right\} \\
D_{2} & =\left\{y_{1}, \ldots, y_{d_{P}-\operatorname{deg}\left(V_{1}\right)},-x_{2(g-1)}\right\} \\
D_{Q} & =\left\{-y_{d_{P}-\operatorname{deg}\left(V_{1}\right)+1}, \ldots,-y_{d_{P}-\operatorname{deg}\left(V_{1}\right)-d_{Q}}\right\} .
\end{aligned}\right.
$$

Let $u$ be the basic epimorphism $[\mathbf{1}]$

$$
u: \operatorname{Div}(X) \longrightarrow \mathrm{H}^{1}\left(X, \mathcal{O}^{*}\right)
$$

and set

$$
\left\{\begin{array}{l}
U_{1}=u\left(D_{1}\right) \\
U_{2}=u\left(D_{2}\right) \\
U_{Q}=u\left(D_{Q}\right) \\
U_{P}=U_{1} \oplus U_{2} .
\end{array}\right.
$$

Let $\psi_{1}, \psi_{2}$ be meromorphic sections associated with the divisors $D_{1}, D_{2}$. Then the meromorphic section $\psi_{1} \oplus \psi_{2}$ of $U_{P}$ is associated with the divisor

$$
D_{1}^{\prime}=\left\{-x_{1}, \ldots,-x_{-\operatorname{deg}\left(U_{1}\right)},-x_{2(g-1)}\right\} .
$$

Hence there exists $V_{1} \subset U_{P}[\mathbf{9}]$ such that

$$
V_{1}=u\left(D_{1}^{\prime}\right)
$$

Let

$$
V_{2}=U_{P} / V_{1}
$$

Since

$$
\begin{gathered}
V_{1} \otimes V_{2}=\operatorname{det}\left(U_{P}\right)=U_{1} \otimes U_{2}, \\
V_{2}=u\left(D_{2}^{\prime}\right),
\end{gathered}
$$

where

$$
D_{2}^{\prime}=\left\{y_{1}, \ldots, y_{d_{P}-\operatorname{deg}\left(V_{1}\right)}\right\} .
$$

In short, the bundle $U_{P}$ is constructed in such a way that it is the trivial extension of $U_{2}$ by $U_{1}$, and is also an extension of $V_{2}$ by $V_{1}$ :

$$
\begin{aligned}
& 0 \longrightarrow U_{1} \stackrel{f_{1}}{\longrightarrow} U_{P} \stackrel{f_{2}}{\longrightarrow} U_{2} \longrightarrow 0 \\
& 0 \longrightarrow V_{1} \stackrel{f_{3}}{\longrightarrow} U_{P} \stackrel{f_{4}}{\longrightarrow} V_{2} \longrightarrow 0 .
\end{aligned}
$$

Tensoring with $\Omega$ gives

$$
\begin{aligned}
& 0 \longrightarrow U_{1} \otimes \Omega \stackrel{g_{1}}{\longrightarrow} U_{P} \otimes \Omega \stackrel{g_{2}}{\longrightarrow} U_{2} \otimes \Omega \longrightarrow 0 \\
& 0 \longrightarrow V_{1} \otimes \Omega \stackrel{g_{3}}{\longrightarrow} U_{P} \otimes \Omega \stackrel{g_{4}}{\longrightarrow} V_{2} \otimes \Omega \longrightarrow 0 .
\end{aligned}
$$


Since

$$
\left\{\begin{aligned}
&-D_{2}+D_{Q}+K^{\prime}=\left\{x_{2(g-1)}, y_{d_{P}-\operatorname{deg}\left(V_{1}\right)-d_{Q}+1}, \ldots, y_{2(g-1)}\right\} \\
&-D_{Q}+D_{1}+K=\left\{y_{d_{P}-\operatorname{deg}\left(V_{1}\right)+1}, \ldots, y_{d_{P}-\operatorname{deg}\left(V_{1}\right)-d_{Q}},\right. \\
&\left.x_{-\operatorname{deg}\left(U_{1}\right)+1}, \ldots, x_{2(g-1)}\right\}
\end{aligned}\right.
$$

are effective divisors, there exists

$$
\begin{aligned}
& 0 \not \equiv \psi_{1} \in \mathrm{H}^{0}\left(X, U_{2}^{-1} \otimes U_{Q} \otimes \Omega\right) \\
& 0 \not \equiv \psi_{2} \in \mathrm{H}^{0}\left(X, U_{Q}^{-1} \otimes U_{1} \otimes \Omega\right) .
\end{aligned}
$$

Set

$$
\Psi_{1}=\psi_{1} \circ f_{2} \text { and } \Psi_{2}=g_{1} \circ \psi_{2} .
$$

Then $\left(U_{1} \oplus U_{2} \oplus U_{Q},\left(\Psi_{1}, \Psi_{2}\right)\right)$ is a semi-stable ternary Hodge bundle.

The divisors

$$
\left\{\begin{aligned}
&-D_{2}^{\prime}+D_{Q}+K^{\prime}=\left\{y_{d_{P}-\operatorname{deg}\left(V_{1}\right)-d_{Q}+1}, \ldots, y_{2(g-1)}\right\} \\
&-D_{Q}+D_{1}^{\prime}+K=\left\{x_{-\operatorname{deg}\left(U_{1}\right)+1}, \ldots, x_{2(g-1)-1},\right. \\
&\left.y_{d_{P}-\operatorname{deg}\left(V_{1}\right)+1}, \ldots, y_{d_{P}-\operatorname{deg}\left(V_{1}\right)-d_{Q}}\right\}
\end{aligned}\right.
$$

are effective. Hence there exist

$$
\begin{aligned}
& 0 \not \equiv \phi_{1} \in \mathrm{H}^{0}\left(X, V_{2}^{-1} \otimes U_{Q} \otimes \Omega\right) \\
& 0 \not \equiv \phi_{2} \in \mathrm{H}^{0}\left(X, U_{Q}^{-1} \otimes V_{1} \otimes \Omega\right) .
\end{aligned}
$$

Remark 2. This is the critical step where the assumption $\tau<2(g-1)$ is needed. In the case of $\tau=2(g-1)$, the degree of $V_{2}^{-1} \otimes U_{Q} \otimes \Omega$ equals -1 thus rendering it impossible to find a non-zero global section $\phi_{1}$. This reflects the fact that every representation with $\tau=2(g-1)$ is reducible. (See Section 6.)

Set

$$
\Psi_{1}^{\prime}=\phi_{1} \circ f_{4} \text { and } \Psi_{2}^{\prime}=g_{3} \circ \phi_{2} .
$$

Then $\left(U_{P} \oplus U_{Q},\left(\Psi_{1}^{\prime}, \Psi_{2}^{\prime}\right)\right)$ is a semi-stable Higgs bundle. Since

$$
\begin{aligned}
& h^{0}\left(X, U_{2}^{-1} \otimes U_{Q} \otimes \Omega\right)>0 \\
& h^{0}\left(X, U_{Q}^{-1} \otimes U_{1} \otimes \Omega\right)>0,
\end{aligned}
$$

$\mathrm{H}^{0}\left(X, U_{1}^{-1} \otimes U_{Q} \otimes \Omega\right)$ and $\mathrm{H}^{0}\left(X, U_{Q}^{-1} \otimes U_{2} \otimes \Omega\right)$ are proper subspaces of $\mathrm{H}^{0}\left(X, U_{P}^{-1} \otimes U_{Q} \otimes \Omega\right)$ and $\mathrm{H}^{0}\left(X, U_{Q}^{-1} \otimes U_{P} \otimes \Omega\right)$, respectively. Hence

$$
\begin{aligned}
F C= & \left(\mathrm{H}^{0}\left(X, U_{P}^{-1} \otimes U_{Q} \otimes \Omega\right) \backslash \mathrm{H}^{0}\left(X, U_{1}^{-1} \otimes U_{Q} \otimes \Omega\right)\right) \times \\
& \left(\mathrm{H}^{0}\left(X, U_{Q}^{-1} \otimes U_{P} \otimes \Omega\right) \backslash \mathrm{H}^{0}\left(X, U_{Q}^{-1} \otimes U_{2} \otimes \Omega\right)\right)
\end{aligned}
$$


is connected and parameterizes a family of semi-stable Higgs bundles that contains both $\left(U_{P} \oplus U_{Q},\left(\Psi_{1}, \Psi_{2}\right)\right)$ and $\left(U_{P} \oplus U_{Q},\left(\Psi_{1}^{\prime}, \Psi_{2}^{\prime}\right)\right)$. Hence there is deformation between the two.

The space of bundle extensions of $V_{2}$ by $V_{1}$,

$$
0 \longrightarrow V_{1} \stackrel{f_{5}}{\longrightarrow} V \stackrel{f_{6}}{\longrightarrow} V_{2} \longrightarrow 0,
$$

is parameterized by the vector space $\mathrm{H}^{1}\left(V_{1} \otimes V_{2}^{-1}\right)$ containing both $U_{P}$ and $V_{1} \oplus V_{2}$ (the zero element in $\mathrm{H}^{1}\left(V_{1} \otimes V_{2}^{-1}\right)$ ). Again tensoring with $\Omega$ gives

$$
0 \longrightarrow V_{1} \otimes \Omega \stackrel{g_{5}}{\longrightarrow} V \otimes \Omega \stackrel{g_{6}}{\longrightarrow} V_{2} \otimes \Omega \longrightarrow 0 .
$$

Let

where

$$
\Phi_{1}=\phi_{1} \circ f_{6}^{\prime} \text { and } \Phi_{2}=g_{5}^{\prime} \circ \phi_{2},
$$

$$
\begin{gathered}
0 \longrightarrow V_{1} \stackrel{f_{5}^{\prime}}{\longrightarrow} V_{1} \oplus V_{2} \stackrel{f_{6}^{\prime}}{\longrightarrow} V_{2} \longrightarrow 0 \\
0 \longrightarrow V_{1} \otimes \Omega \stackrel{g_{5}^{\prime}}{\longrightarrow}\left(V_{1} \oplus V_{2}\right) \otimes \Omega \stackrel{g_{6}^{\prime}}{\longrightarrow} V_{2} \otimes \Omega \longrightarrow 0
\end{gathered}
$$

correspond to the trivial extensions. By Lemma $7.4, \mathrm{H}^{1}\left(V_{1} \otimes V_{2}^{-1}\right)$ parameterizes a family of semi-stable Higgs bundles that contains both $\left(U_{P} \oplus\right.$ $\left.U_{Q},\left(\Psi_{1}^{\prime}, \Psi_{2}^{\prime}\right)\right)$ and $\left(V_{1} \oplus V_{2} \oplus U_{Q},\left(\Phi_{1}, \Phi_{2}\right)\right)$.

To summarize, the first step consists of fixing $U_{P}=U_{1} \oplus U_{2}$ and deform the Higgs field $\left(\Psi_{1}, \Psi_{2}\right)$ to $\left(\Psi_{1}^{\prime}, \Psi_{2}^{\prime}\right)$. In the second step, fix $\phi_{1}, \phi_{2}$ and deform $U_{P}$ to $V_{1} \oplus V_{2}$.

Consider the space $T\left(0, d_{2},-d_{2}\right)$. By Proposition 7.5 , one may assume $d_{2}>$ 0 . To deform points in $T\left(0, d_{2},-d_{2}\right)$, the family $F C$ constructed in the above proof contains semi-stable Higgs bundles. However, one may also opt to construct the deformation family of poly-stable Higgs bundles by setting:

$$
\begin{aligned}
F C= & \left(\mathrm{H}^{0}\left(X, U_{P}^{-1} \otimes U_{Q} \otimes \Omega\right) \backslash\right. \\
& \left.\left(\mathrm{H}^{0}\left(X, U_{1}^{-1} \otimes U_{Q} \otimes \Omega\right) \cup \mathrm{H}^{0}\left(X, U_{2}^{-1} \otimes U_{Q} \otimes \Omega\right)\right)\right) \times \\
& \left(\mathrm{H}^{0}\left(X, U_{Q}^{-1} \otimes U_{P} \otimes \Omega\right) \backslash\right. \\
& \left.\left(\mathrm{H}^{0}\left(X, U_{Q}^{-1} \otimes U_{2} \otimes \Omega\right) \cup \mathrm{H}^{0}\left(X, U_{Q}^{-1} \otimes U_{1} \otimes \Omega\right)\right)\right) \\
& \cup\left(\mathrm{H}^{0}\left(X, U_{2}^{-1} \otimes U_{Q} \otimes \Omega\right) \times\{0\}\right) .
\end{aligned}
$$

The case with $\tau=2(g-1)$ has been covered in Section 6 and $\mathcal{M}_{\mathrm{Dol}}^{2(g-1)}$ is connected. Suppose $\tau<2(g-1)$. By Proposition 7.8, every component of $\mathcal{M}_{\text {Dol }}^{\tau}$ contains a Hodge bundle. By Corollary 7.6, every component of $\mathcal{M}_{\text {Dol }}^{\tau}$ contains a ternary Hodge bundle. It follows from Proposition 7.10 and induction that $\mathcal{M}_{\text {Dol }}^{\tau}$ is connected. Since

$$
\mathcal{N}_{\text {Dol }}^{\tau}=\mathcal{M}_{\text {Dol }}^{\tau} / \mathrm{H}^{1}\left(X, \mathcal{O}^{*}\right),
$$

Theorem 1.1 then follows from Corollary 4.2. 


\section{References}

[1] E. Arbarello, M. Cornalba, P. Griffiths and J. Harris, Geometry of Algebraic Curves, Vol. I, Springer-Verlag, 1985.

[2] M. Atiyah and R. Bott, The Yang-Mills equations over Riemann surfaces, Philos. Trans. Roy. Soc. London, Ser., A 308 (1982), 523-615.

[3] K. Corlette, Flat G-bundles with canonical metrics, J. Diff. Geom., 28 (1988), 361382 .

[4] A. Domic and D. Toledo, The Gromov norm of the Kähler class of symmetric domains, Math. Ann., 276 (1987), 425-432.

[5] S. Donaldson, Twisted harmonic maps and the self-duality equations, Proc. London Math. Soc., 55 (1987), 127-131.

[6] W. Goldman, Topological components of spaces of representations, Invent. Math., 93 (1988), 557-607.

[7] W. Goldman, Michael E. Kapovich and Bernhard Leeb, Complex Hyperbolic Manifolds Homotopy-Equivalent to a Riemann Surface, Preprint.

[8] P. Gothen, The Betti numbers of the moduli space of stable rank 3 Higgs bundles on a Riemann surface, Internat. J. Math., 5(6) (1994), 861-875.

[9] R. Gunning, Lectures on Vector Bundles Over Riemann Surfaces, Princeton University Press, 1967.

[10] R. Hartshorne, Algebraic Geometry, Springer-Verlag, 1977.

[11] N. Hitchin, The self-duality equations on a Riemann surface, Proc. London Math. Soc., 55 (1987), 59-126.

[12] _ Lie groups and Teichmüller space, Topology, 31 (1992), 449-473.

[13] H. Lange and M. Narasimhan, Maximal subbundles of rank two vector bundles on curves, Math. Ann., 266 (1983), 55-72.

[14] M. Maruyama, Openness of a family of torsion free sheaves, J. Math. Kyoto Univ., 16-3 (1976), 627-637.

[15] D. Mumford and J. Fogarty, Geometric Invariant Theory, 2nd edn., Springer, Berlin, 1982 .

[16] M. Narasimhan and C. Seshadri, Stable and unitary vector bundles on a compact Riemann surface, Annals of Math., 82 (1965), 540-567.

[17] P.E. Newstead, Introductions to Moduli Problems and Orbit Spaces, Springer-Verlag, 1978.

[18] N. Nitsure, Moduli space of semistable pairs on a curve, Proc. London Math. Soc., 62 (1991), 275-300.

[19] C. Seshadri, Fibrés vectoriels sur les courbes algebriques, Astérisque, 96 (1982).

[20] S. Shatz, The decomposition and specialization of algebraic families of vector bundles, Compositio Mathematica, 35 (1977), 163-187.

[21] C. Simpson, Constructing variations of Hodge structures using Yang-Mills Theory and applications to uniformization, Journal of the A.M.S., 1 (1988), 867-918.

[22] _ Higgs bundles and local systems, Publ. Math. I.H.E.S., 75 (1992), 6-95.

[23] _ Moduli of representations of the fundamental group of a smooth projective variety, I, Publ. Math. I.H.E.S., 79 (1994), 47-129. 
[24] _ Moduli of representations of the fundamental group of a smooth projective variety, II, Publ. Math. I.H.E.S., 80 (1994), 5-79.

[25] N. Steenrod, The Topology of Fiber Bundles, Princeton University Press, 1951.

[26] D. Toledo, Harmonic maps from surfaces to certain Kähler manifolds, Math. Scand., 45 (1979), 13-26.

[27] _ Representations of surface groups in complex hyperbolic space, J. Differential Geometry, 29 (1989), 125-133.

Received September 2, 1998 and revised March 16, 1999.

University of MASSACHUSETtS

AmHerst, MA 01003-4515

E-mail address: xia@math.umass.edu 